TRANSACTIONS OF THE

AMERICAN MATHEMATICAL SOCIETY

Volume 357, Number 8, Pages 3375-3405

S 0002-9947(05)03696-2

Article electronically published on March 25, 2005

\title{
AN APPROXIMATE UNIVERSAL COEFFICIENT THEOREM
}

\author{
HUAXIN LIN
}

\begin{abstract}
An approximate Universal Coefficient Theorem (AUCT) for certain $C^{*}$-algebras is established. We present a proof that Kirchberg-Phillips's classification theorem for separable nuclear purely infinite simple $C^{*}$-algebras is valid for $C^{*}$-algebras satisfying the AUCT instead of the UCT. It is proved that two versions of AUCT are in fact the same. We also show that $C^{*}$-algebras that are locally approximated by $C^{*}$-algebras satisfying the AUCT satisfy the AUCT. As an application, we prove that certain simple $C^{*}$-algebras which are locally type I are in fact isomorphic to simple AH-algebras. As another application, we show that a sequence of residually finite-dimensional $C^{*}$-algebras which are asymptotically nuclear and which asymptotically satisfies the AUCT can be embedded into the same simple AF-algebra.
\end{abstract}

\section{INTRODUCTION}

The Universal Coefficient Theorem for $C^{*}$-algebras was first introduced by L. G. Brown in the connection with the study of extensions of $C^{*}$-algebras $([\mathrm{Br}])$. A $C^{*}$-algebra, $A$ satisfies the Universal Coefficient Theorem (UCT) if for any $\sigma$-unital $C^{*}$-algebra, $B$, one has the following short exact sequence:

$$
0 \rightarrow \operatorname{Ext}_{\mathbb{Z}}\left(K_{*}(A), K_{*}(B)\right) \stackrel{\delta}{\rightarrow} K K^{*}(A, B) \stackrel{\gamma}{\rightarrow} \operatorname{Hom}\left(K_{*}(A), K_{*}(B)\right) \rightarrow 0,
$$

where $\gamma$ has degree 0 and $\delta$ has degree 1. It was shown by Rosenberg and Schochet $[\mathrm{RS}]$ that every $C^{*}$-algebra, in the so-called "bootstrap" class satisfies the Universal Coefficient Theorem (UCT). It is a very important tool in $K K$-theory. It was pointed out by M. Rørdam (see [Rr2]) that a quotient of $K K$, namely $K L$, is more relevant in the theory of classification of nuclear $C^{*}$-algebras and perhaps in many other applications. Therefore it might be possible that a version of the UCT for $K L$ would suffice for many important purposes. However, we need to define $K L$ without using the UCT. Moreover, without the UCT, one needs to establish a number of facts before one can effectively use the functor $K L$.

In this paper, we will present an approximate version of the Universal Coefficient Theorem. We will show that such an approximate version of UCT for KL is indeed sufficient for many purposes, in particular, for the classification of nuclear simple $C^{*}$-algebras.

There are two possible approximate versions of UCT for KL. When $A$ satisfies the UCT, stably approximately trivial extensions of $A$ by $S B$ are represented by $\operatorname{Pext}\left(K_{*}(A), K_{*-1}(B)\right)$, the pure extensions of the abelian groups. If

Received by the editors October 1, 2002 and, in revised form, March 12, 2004.

2000 Mathematics Subject Classification. Primary 46L05, 46L35, 46L80.

Key words and phrases. Approximate universal coefficient theorem.

This research was partially supported by NSF grants DMS 0097903 and 0355273. 
$E_{0}\left(K_{*}(A), K_{*-1}(B)\right)$ denotes the quotient

$$
\operatorname{Ext}_{\mathbb{Z}}\left(K_{*}(A), K_{*-1}(B)\right) / \operatorname{Pext}\left(K_{*}(A), K_{*-1}(B)\right),
$$

one possible version of the approximate $\mathrm{UCT}$ is

$$
0 \rightarrow E_{0}\left(K_{*}(A), K_{*-1}(B)\right) \rightarrow K L(A, B) \rightarrow \operatorname{Hom}\left(K_{*}(A), K_{*}(B)\right) \rightarrow 0 .
$$

A more often used UCT in classification theory is the Dadarlat-Loring multicoefficient UCT:

$$
0 \rightarrow \operatorname{Pext}\left(K_{*}(A), K_{*-1}(B)\right) \rightarrow K K(A, B) \rightarrow \operatorname{Hom}_{\Lambda}(\underline{K}(A), \underline{K}(B)) \rightarrow 0 .
$$

The Dadarlat-Loring's UCT plays an increasingly important role in the study of classification of nuclear $C^{*}$-algebras. Correspondingly another approximate UCT for $K L$ is

$$
K L(A, B)=\operatorname{Hom}_{\Lambda}(\underline{K}(A), \underline{K}(B)) .
$$

In the absence of the UCT, we will show these two versions of approximate UCT for $K L$ are in fact equivalent at least for separable nuclear $C^{*}$-algebras. This enables us to define the approximate UCT (AUCT) for $K L$. Of course $C^{*}$-algebras satisfying the UCT also satisfy the AUCT. At this moment, it is not clear that the AUCT and UCT are the same in general. Nevertheless, our results do show that, for example, a separable nuclear purely infinite simple $C^{*}$-algebra, that satisfies the AUCT satisfies the UCT. In section 3 , in the absence of UCT, we strengthen our earlier uniqueness theorem for homomorphisms using $K L$. It is clear that $K L(A, B)$ should be the right tool for stably approximately unitarily equivalent homomorphisms (from $A$ to $B$ ). However, a general version of this uniqueness theorem was not obtained earlier. With this uniqueness theorem, we show that for classification of separable nuclear $C^{*}$-algebras, only $K L$ is needed. We then present a proof that the Kirchberg-Phillips classification theorem for separable nuclear purely infinite simple $C^{*}$-algebras works under the assumption that these $C^{*}$-algebras satisfy the AUCT (instead of UCT). This also applies to the case that $C^{*}$-algebras have tracial topological rank zero.

This may lead one to speculate that perhaps more nuclear $C^{*}$-algebras satisfy the approximate $\mathrm{UCT}$ for $K L$, or at least the approximate $\mathrm{UCT}$ for $K L$ may be somewhat easier to establish.

It was shown by Schochet ([S1] $)$, using L. G. Brown's mapping telescope construction, that if $A=\lim _{n}\left(A_{n}, h_{n}\right)$, where $A_{n}$ are separable $C^{*}$-algebras satisfying the UCT and $h_{n}$ are monomorphisms, then $A$ satisfies the UCT. Suppose that $A$ is a $C^{*}$-algebra, which satisfies the following local approximation property: for any $\varepsilon>0$ and any finite subset $\mathcal{F}$, there is a $C^{*}$-subalgebra $B$ of $A$ which satisfies the AUCT such that

$$
\operatorname{dist}(x, B)<\varepsilon \text { for all } x \in \mathcal{F} \text {. }
$$

$C^{*}$-algebras satisfying this kind of local approximation property have appeared more often recently. It was shown in DE1 that there are $C^{*}$-algebras which can be locally approximated by homogeneous $C^{*}$-algebras but are not expressible as inductive limits of homogeneous $C^{*}$-algebras. The question is whether a $C^{*}$-algebra that has the above-mentioned local approximation property satisfies the approximate UCT for KL. Another purpose of this paper is to answer this question (see 6.16 and 6.17).

Let $A$ be a unital separable simple $C^{*}$-algebra, with real rank zero, stable rank one, weakly unperforated $K_{0}(A)$ and with a unique tracial state. It is shown in 
Ln9 that, if, in addition, $A$ is locally approximable by type I $C^{*}$-algebras, then the tracial topological rank of $A$ is zero. However, in order to apply the classification theorem in [Ln8, one needs another assumption: $A$ satisfies the UCT. It is easy to see that $A$ is nuclear. By 6.16, $A$ automatically satisfies the AUCT. We will also show that the classification theorem in $\operatorname{Ln} 8$ holds for $C^{*}$-algebras satisfying the AUCT (instead of the UCT) (see 5.10). Thus the classification theorem (5.10) can also be applied to the above mentioned $C^{*}$-algebra $A$. This is one of our original motivations for establishing the AUCT. As another application, we show that a sequence of residually finite-dimensional $C^{*}$-algebras which is asymptotically nuclear and asymptotically satisfies the (A)UCT can be embedded into one AF-algebra (see (7.8).

\section{The FUnCTOR $K L(-,-)$}

We use the following notation:

(i) Let $B$ be a $C^{*}$-algebra. In what follows, we denote by $S B$ the suspension of $B$, i.e., $S B=C_{0}((-1,1), B)$.

(ii) Let $\mathcal{K}$ be the $C^{*}$-algebra of compact operators on $l^{2}$. Let $B$ be a $C^{*}$-algebra. We denote by $M(B)$ the multiplier algebra of $B$. We denote by $Q(B)$ the stable quotient $M(B \otimes \mathcal{K}) / B \otimes \mathcal{K}$.

(iii) Denote by $\mathcal{N}$ the so called "bootstrap" class of $C^{*}$-algebras (see [RS]).

(iv) For each $n$, set

$$
\mathbb{I}_{n}=\left\{f \in C\left([0,1], M_{n}\right): f(0)=0 \quad \text { and } \quad f(1) \in \mathbb{C} \cdot 1\right\} .
$$

It follows from Lo2 that $\mathbb{I}_{n}$ is a semiprojective $C^{*}$-algebra.

(v) Let $\mathbb{Q}$ be the field of rational numbers. Denote by $Q$ the UHF-algebra with $K_{0}(Q)=\mathbb{Q}$ and $\left[1_{Q}\right]=1$.

Definition 2.1. Let $A$ be a nuclear $C^{*}$-algebra, and let $B$ be a $\sigma$-unital $C^{*}$-algebra. Let

$$
0 \rightarrow B \rightarrow E \rightarrow A \rightarrow 0
$$

be an extension and let $\tau: A \rightarrow M(B) / B$ be the Busby invariant. Recall that the above extension is said to be trivial if the extension splits. Let $\pi: M(B) \rightarrow$ $M(B) / B$ be the quotient map. Then if $E$ is trivial, there is homomorphism $\sigma$ : $A \rightarrow M(B)$ such that $\tau=\pi \circ \sigma$.

An extension $\tau: A \rightarrow M(B) / B$ of $A$ by $B$ determined by

$$
0 \rightarrow B \rightarrow E \rightarrow A \rightarrow 0
$$

is said to be approximately trivial if there are trivial extensions $\tau_{n}: A \rightarrow M(B) / B$ such that

for every $a \in A$.

$$
\left\|\tau_{n}(a)-\tau(a)\right\| \rightarrow 0 \quad \text { as } n \rightarrow \infty
$$

Recall that an extension $\tau: A \rightarrow Q(B)$ determined by

$$
0 \rightarrow B \otimes \mathcal{K} \rightarrow E \rightarrow A \rightarrow 0
$$

is stably trivial if $\tau \oplus \tau_{0}$ is trivial for some trivial extension $\tau_{0}: A \rightarrow Q(B)$. We say $\tau$ is stably approximately trivial if there is a trivial extension $\tau_{0}$ such that $\tau \oplus \tau_{0}$ is approximately trivial.

Two extensions $\tau_{1}$ and $\tau_{2}$ are stably unitarily equivalent if there is a trivial extension $\tau_{0}$ and there is a unitary $u \in Q(B)$ such that $u^{*}\left(\tau_{1} \oplus \tau_{0}\right) u=\tau_{2} \oplus \tau_{0}$. 
An extension $\tau$ is absorbing if $\tau \oplus \tau_{0}$ is unitarily equivalent to $\tau$ for all trivial extensions $\tau_{0}$. Denote by $\operatorname{Ext}(A, B)$ the set of stable unitary equivalence classes of extensions of $A$ by $B \otimes \mathcal{K}$. It becomes a semigroup with zero element represented by stably trivial extensions. Denote by $\operatorname{Ext}^{-1}(A, B)$ the set of invertible elements in $\operatorname{Ext}(A, B)$. It is known (see $[\mathrm{A}]$ and $[\mathrm{CE}])$ that $\operatorname{Ext}(A, B)$ is a group, provided $A$ is nuclear. Denote by $\mathcal{T}(A, B)$ the set of equivalence classes of stably approximately trivial extensions.

As in Schochet ([S4), there is a metric on $\operatorname{Ext}(A, B)$, and $\mathcal{T}(A, B)$ is precisely the closure of the zero (stably trivial extensions) element in that topology.

Lemma 2.2. Let $A$ and $B$ be $\sigma$-unital $C^{*}$-algebras. The subset

$$
\mathcal{T}(A, B) \cap \operatorname{Ext}^{-1}(A, B)
$$

is a group.

Proof. It is clear that the sum of two stably approximately trivial extensions is again a stably approximately trivial extension. Thus $\mathcal{T}(A, B)$ is closed under the addition. Let $y \in \operatorname{Ext}^{-1}(A, B)=K K^{1}(A, B)$. Suppose that $x \in K K(A, A)$ is the inverse of $\left[\operatorname{id}_{A}\right]$ in $K K(A, A)$. Then $x \times y=-y$. It follows from a result of Schochet (see [S3] ) that the Kasparov product is continuous with respect to the Salinas topology. Therefore, if $y \in \mathcal{T}(A, B)$ which is in the closure of the zero, then $x \times y \in \mathcal{T}(A, B)$. Therefore $-y \in \mathcal{T}(A, B)$.

Definition 2.3. Let $B$ be a $\sigma$-unital $C^{*}$-algebra. We identify $K K(A, B)$ with $K K^{1}(S A, B)=\operatorname{Ext}^{-1}(S A, B)$. We follow the notation first introduced by Rørdam $([\mathrm{Rr} 2])$. Let $B$ be a $\sigma$-unital $C^{*}$-algebra. Define

$$
K L(A, B)=\operatorname{Ext}^{-1}(S A, B) / \operatorname{Ext}^{-1}(S A, B) \cap \mathcal{T}(S A, B) .
$$

Let $\Pi: K K(A, B) \rightarrow K L(A, B)$ be the quotient map.

It should be noted that previously $K L(A, B)$ was only defined for $C^{*}$-algebras satisfying the UCT since its definition depended on the UCT (a slightly more general definition using pure extensions also appeared). Here we define $K L$ without assuming that $A$ satisfies the UCT nor do we assume that $A$ is nuclear (even though we are mostly interested in the case that $A$ is nuclear). Therefore, to effectively use $K L$, we need to establish certain facts without the UCT.

Let $f: A \rightarrow B$ be a homomorphism. Let $f_{1}: S A \rightarrow S B$ be the induced homomorphism. For each $x \in \operatorname{Ext}^{-1}(S B, D)$, define $f^{*}(x)=\left[\tau \circ f_{1} \oplus t\right]$, where $[\tau]=x$ and $t: A \rightarrow Q(D)$ is a trivial extension. We see that $f^{*}(\mathcal{T}(S B, D)) \subset$ $\mathcal{T}(S A, D)$. Define $f^{*}(\Pi(x))=\Pi\left(f^{*}(x)\right)$. This is well defined. From this we see that $K L(-, D)$ is a contravariant functor from $\sigma$-unital $C^{*}$-algebras to abelian groups.

Let $f: B \rightarrow D$ be a homomorphism, where both $B$ and $D$ are assumed to be $\sigma$-unital. If $B$ is unital, then $f$ extends uniquely a homomorphism $\tilde{f}: M(B \otimes \mathcal{K}) \rightarrow$ $M(D \otimes \mathcal{K})$. Define $\bar{f}: Q(B) \rightarrow Q(D)$ to be the induced map. For $x \in \operatorname{Ext}^{-1}(S A, B)$, we define $f_{*}(x)=[\bar{f} \circ \tau \oplus t]$, where $[\tau]=x$ and $t$ is a trivial extension. If $B$ is not unital, let $\tilde{f}: \tilde{B} \rightarrow \tilde{D}$ be the extension. We still use $\tilde{f}$ for the extension from $\tilde{B} \otimes \mathcal{K}$ to $\tilde{D} \otimes \mathcal{K}$. Note that both $\tilde{B} \otimes \mathcal{K}$ and $\tilde{D} \otimes \mathcal{K}$ are $\sigma$-unital. $\tilde{f}$ extends to a homomorphism from $M(\tilde{B} \otimes \mathcal{K})$ to $M(\tilde{D} \otimes \mathcal{K})$. We identify $M(\tilde{B} \otimes \mathcal{K})$ and $M(\tilde{D} \otimes \mathcal{K})$ with the $C^{*}$-subalgebra of $M(B \otimes \mathcal{K})$ and $M(D \otimes \mathcal{K})$, respectively. Thus $\tilde{f}$ induces a homomorphism $\bar{f}: M(\tilde{B} \otimes \mathcal{K}) / B \otimes \mathcal{K} \rightarrow M(\widetilde{D} \otimes \mathcal{K}) / D \otimes \mathcal{K}$. It follows from 1.4 
in [H] that there is an isometry $v \in M(B \otimes \mathcal{K})$ such that

$$
v M(B \otimes \mathcal{K}) v^{*} \subset M(\tilde{B} \otimes \mathcal{K}) .
$$

Thus, for any $[\tau] \in \operatorname{Ext}(S A, B), v \tau(a) v^{*} \in M(\tilde{B} \otimes \mathcal{K})$. We define $f_{*}([\tau])=$ $\left[\bar{f} \circ v \tau v^{*}\right]$. From the definition of $f_{*}$, it is clear that $f_{*}(\mathcal{T}(S A, B)) \subset \mathcal{T}(S A, D)$. Define $f_{*}(\Pi(x))=\Pi\left(f_{*}(x)\right)$. Then, $K L(A,-)=\operatorname{Ext}^{-1}(S A,-)$ becomes a covariant functor from $\sigma$-unital $C^{*}$-algebras to abelian groups. If we use the fact that $f_{*}(x)=x \times[f]$, then it also easily follows from the result of Schochet (see [S3]) that the Kasparov product is continuous.

Since $f_{*}$ and $f^{*}$ both map the closure of the zero to the closure of zero, from the fact that $K K(-, A)$ and $K K(A,-)$ are homotopy invariant, stable and split exact, we obtain the following:

Proposition 2.4. (1) If $A$ is a separable $C^{*}$-algebra, then $K L(A,-)$ is a homotopy invariant, stable and split exact covariant functor from $\sigma$-unital $C^{*}$-algebras to abelian groups.

(2) If $A$ is a $\sigma$-unital $C^{*}$-algebra, then $K L(-, A)$ is a homotopy invariant, stable and split exact covariant functor from separable $C^{*}$-algebras to abelian groups.

Definition 2.5. Let $A$ be a $\sigma$-unital nuclear $C^{*}$-algebra and let $B$ be a $\sigma$-unital $C^{*}$-algebra. We define $\operatorname{Ext}_{a p}(A, B)$ to be the quotient $\operatorname{Ext}(A, B) / \mathcal{T}(A, B)$.

Remark 2.6. The set $\mathcal{T}(A, B)$ is the same as the closure of zero in $\operatorname{Ext}(A, B)$ in [S3]. Schochet (Theorem 3.1 in $[\mathrm{S} 3$ ) showed that the Kasparov product is continuous. Thus, one can define the Kasparov product on $K L$. His proof works for the nonnuclear case, too.

\section{Some Uniqueness theOREMS}

Definition 3.1. For any $\sigma$-unital $C^{*}$-algebra, $A$, there is an embedding $A \rightarrow A \otimes \mathcal{K}$ defined by $a \mapsto a \otimes e_{11}$, where $\left\{e_{i j}\right\}$ is a fixed system of matrix units for $\mathcal{K}$. One can then identify $M(A)$ with $\left(1 \otimes e_{11}\right) M(A \otimes \mathcal{K})\left(1 \otimes e_{11}\right)$ which gives an embedding $M(A) \rightarrow M(A \otimes \mathcal{K})$. Let $A$ be a $C^{*}$-algebra, and let $B$ be a $\sigma$-unital stable $C^{*}$ algebra. We set $C=\prod_{n=1}^{\infty} B$ and $C_{0}=\bigoplus_{n=1}^{\infty} B$, the product and direct sum of a sequence of $C^{*}$-algebras each of which is $B$, respectively. We obtain a map $\phi_{1}: M(C) \rightarrow M(C \otimes \mathcal{K})$. We will identify $M\left(\prod_{n=1}^{\infty} B\right)$ with $\prod_{n=1}^{\infty} M(B)$. Let $\phi_{2}: C \otimes \mathcal{K} \rightarrow\left(C / C_{0}\right) \otimes \mathcal{K}$ be the quotient map. Then (by [Ped1]) there is a (surjective) homomorphism, again denoted by $\phi_{2}$, from $M(C \otimes \mathcal{K})$ to $M\left(\left(C / C_{0}\right) \otimes \mathcal{K}\right)$ which extends $\phi_{2}$. Put $\phi=\phi_{2} \circ \phi_{1}$. We will use $\pi$ for both quotient maps from $M(C)$ to $M\left(C / C_{0}\right)$ and from $M\left(\left(C / C_{0}\right) \otimes \mathcal{K}\right)$ to $M\left(\left(C / C_{0}\right) \otimes \mathcal{K}\right) /\left(\left(C / C_{0}\right) \otimes \mathcal{K}\right)$.

We have the following lemma:

\section{Lemma 3.2.}

$$
\bigoplus_{n=1}^{\infty} M(B) \subset \operatorname{ker} \phi
$$

and

$$
\bigoplus_{n=1}^{\infty} M(B)+\prod_{n=1}^{\infty} B \subset \operatorname{ker} \pi \circ \phi
$$


Proof. Let $b \in \bigoplus_{n=1}^{\infty} M(B)$. We need to show that $\phi_{1}(b) c, c \phi_{1}(b) \in\left(\bigoplus_{n=1}^{\infty} B\right) \otimes \mathcal{K}$ for any $c \in\left(\prod_{n=1}^{\infty} B\right) \otimes \mathcal{K}$. Since we may assume that $b$ is self-adjoint, we only need to show that $\phi_{1}(b) c \in \bigoplus_{n=1}^{\infty} B$. The element $\phi(b)$ may be identified with an infinite matrix whose only non-zero entry is $b$ at the $(1,1)$ position. Write $c=\left(c_{i j}\right)$, where $c_{i j} \in \prod_{n=1}^{\infty} B$. Then $\phi_{1}(b) c=\left(d_{i j}\right)$, where $d_{i j}=0$ if $i \neq 1$ and $d_{1 j}=b c_{1 j}$ for all $j$. Since $b \in \bigoplus_{n=1}^{\infty} M(B)$, each $d_{1 j} \in \bigoplus_{n=1}^{\infty} B$. Since $c \in\left(\prod_{n=1}^{\infty} B\right) \otimes \mathcal{K}$, one obtains that $\left\|\sum_{j=n}^{\infty} c_{1 j}^{*} c_{1 j}\right\| \rightarrow 0$ as $n \rightarrow \infty$. This implies that

$$
\left\|\sum_{j=n}^{\infty} d_{1 j}^{*} d_{1 j}\right\| \leq\|b\|^{2}\left\|\sum_{j=n}^{\infty} c_{1 j}^{*} c_{1 j}\right\| \rightarrow 0 \text { as } n \rightarrow \infty .
$$

Therefore $\left(d_{i j}\right) \in\left(\bigoplus_{n=1}^{\infty} B\right) \otimes \mathcal{K}$.

The second inclusion holds because $\phi_{1}$ maps $\prod_{n=1}^{\infty} B$ to $\left(\prod_{n=1}^{\infty} B\right) \otimes \mathcal{K}$ and $\phi_{2}$ maps $\left(\prod_{n=1}^{\infty} B\right) \otimes \mathcal{K}$ to $\left(\prod_{n=1}^{\infty} B / \bigoplus_{n=1}^{\infty} B\right) \otimes \mathcal{K}$.

Definition 3.3. Let $B$ be a $\sigma$-unital stable $C^{*}$-algebra, and let $A$ be a nuclear $C^{*}$-algebra. Let $\tau: A \rightarrow M(B) / B$ be an extension of $A$ by $B$. Denote by $\pi_{0}$ : $M(B) \rightarrow M(B) / B$ the quotient map. Define $j: B \rightarrow \prod_{n=1}^{\infty} B$ by $j(b)=(b, b, \ldots$, (the constant sequence). Again, we let $C=\prod_{n=1}^{\infty} B$ and $C_{0}=\bigoplus_{n=1}^{\infty} B$. Let $\pi_{1}: C \rightarrow C / C_{0}$ be the quotient map. The map $j$ extends to a map from $M(B)$ to $\prod_{n=1}^{\infty} M(B)=M\left(\prod_{n=1}^{\infty} B\right)$. Put $\Psi=\pi_{1} \circ j$. Then $\Psi$ is a monomorphism from $B$ to $C / C_{0}$. The map $\pi_{1}$ can be extended to a (surjective) map from $M\left(\prod_{n=1}^{\infty} B\right)$ to $M\left(C / C_{0}\right)$. Thus we obtain an extension of $\Psi$ which maps $\tilde{\Psi}: M(B) \rightarrow M\left(C / C_{0}\right)$. Moreover, as in 3.1, $\Psi$ can also be extended to $\tilde{\Psi}: M(B) \rightarrow M\left(\left(C / C_{0}\right) \otimes \mathcal{K}\right)$. Denote by $\bar{\Psi}$ the induced map from $M(B) / B$ to $M\left(C / C_{0}\right) /\left(C / C_{0}\right)$ (and the induced map from $M(B) / B$ to $\left.M\left(\left(C / C_{0}\right) \otimes \mathcal{K}\right) /\left(\left(C / C_{0}\right) \otimes \mathcal{K}\right)\right)$. With the above notation, $\Psi_{*}([\tau])=[\tilde{\Psi} \circ \tau]$. Suppose that $\phi^{\prime}: A \rightarrow M(B)$ is a contractive completely positive linear map such that $\pi_{0} \circ \phi=\tau$. With notation in [3.1, we also have $[\bar{\Psi} \circ \tau]=$ $\left[\pi \circ \phi_{2} \circ \phi_{1} \circ j \circ \varphi\right]$, where $\pi: M\left(\left(C / C_{0}\right) \otimes \mathcal{K}\right) \rightarrow M\left(\left(C / C_{0}\right) \otimes \mathcal{K}\right) /\left(\left(C / C_{0}\right) \otimes \mathcal{K}\right)$ is the quotient map. In other words, $\Psi_{*}([\tau])=[\tilde{\Psi} \circ \tau]=\left[\pi \circ \phi_{2} \circ \phi_{1} \circ j \circ \varphi\right]$.

Consider $\Psi_{*}: \operatorname{Ext}(A, B) \rightarrow \operatorname{Ext}\left(A, \prod_{n=1}^{\infty} B / \bigoplus_{n=1}^{\infty} B\right)$. Using the Kasparov product, we have $\Psi_{*}([\tau])=[\tau] \times[\Psi]$ (see, for example, 18.7.2 in $[\mathrm{B}]$ ), where $[\Psi]$ is viewed as an element in $K K\left(B, \prod_{n=1}^{\infty} B / \bigoplus_{n=1}^{\infty} B\right)$.

Theorem 3.4. Let $A$ be a separable nuclear $C^{*}$-algebra and let $B$ be a $\sigma$-unital stable $C^{*}$-algebra. Then

$$
\mathcal{T}(A, B) \subset \operatorname{ker} \Psi_{*} .
$$

Proof. Suppose that $\tau: A \rightarrow M(B) / B$ is an extension in $\mathcal{T}(A, B)$. Let $\phi: A \rightarrow$ $M(B)$ be a contractive completely positive linear map such that $\pi \circ \phi=\tau$, where $\pi: M(B) \rightarrow M(B) / B$. By adding an absorbing trivial extension, if necessary, we may assume that there is a sequence of trivial extensions $\tau_{n}: A \rightarrow M(B) / B$ such that

$$
\lim _{n \rightarrow \infty} \tau_{n}(a)=\tau(a) \text { for all } a \in A
$$

Let $h_{n}: A \rightarrow M(B)$ be a monomorphism such that $\pi \circ h_{n}=\tau_{n}$, where $\pi$ : $M(B) \rightarrow M(B) / B$ is the quotient map. Let $H: A \rightarrow \prod_{n=1}^{\infty} M(B)$ be defined by $H(a)=\left(h_{1}(a), \ldots, h_{n}(a), \ldots\right)$. Then, with notation as in 3.3 ,

$$
H(a)-j \circ \varphi(a) \in \bigoplus_{n=1}^{\infty} M(B)+\prod_{n=1}^{\infty} B_{n}
$$


for all $a \in A$. Then it follows from 3.2 that

$$
\pi \circ \phi_{1} \circ \phi_{2}(H(a)-j \circ \varphi(a))=0 \text { for all } a \in A .
$$

This implies that

$$
\Psi_{*}([\tau])=\left[\pi \circ \phi_{1} \circ \phi_{2} \circ H\right]
$$

Since $\phi_{1} \circ \phi_{2} \circ H$ is a homomorphism from $A$ to $M\left(\left(\prod_{n=1}^{\infty} B / \bigoplus_{n=1}^{\infty} B\right) \otimes \mathcal{K}\right)$, we have that $\pi \circ \phi_{1} \circ \phi_{2} \circ H$ is trivial. Therefore

$$
\Psi_{*}([\tau])=0 .
$$

Corollary 3.5. Let $A$ be a separable nuclear $C^{*}$-algebra, and let $B$ be a $\sigma$-unital $C^{*}$-algebra. Suppose that $h_{i}: A \rightarrow B$ are two homomorphisms $(i=1,2)$ such that $\left[h_{1}\right]=\left[h_{2}\right]$ in $K L(A, B)$. Let $H_{i}: A \rightarrow \prod_{n=1}^{\infty} B$ be the homomorphism defined by $H_{i}(a)=\left(h_{i}(a), h_{i}(a), \ldots, h_{i}(a), \ldots\right), i=1,2$, and let $\pi_{1}: \prod_{n=1}^{\infty} B \rightarrow$ $\prod_{n=1}^{\infty} B / \bigoplus_{n=1}^{\infty} B$ be the quotient map. Then

$$
\left[\pi_{1} \circ H_{1}\right]=\left[\pi_{1} \circ H_{2}\right] \text { in } K K\left(A, \prod_{n=1}^{\infty} B / \bigoplus_{n=1}^{\infty} B\right) \text {. }
$$

Proof. We identify $K L(A, B)$ with $\operatorname{Ext}_{a p}(S A, B)$. Let $\left[h_{i}\right]$ be the image of $h_{i}$ in $\operatorname{Ext}_{a p}(S A, B)$. Note that $\pi_{1} \circ H_{i}=\pi_{1} \circ j \circ h_{i}=\Psi \circ h_{i}$ for $i=1,2$. Then $\left[\pi_{1} \circ H_{i}\right]=$ $\Psi_{*}\left(\left[h_{i}\right]\right)=\left[h_{i}\right] \times[\Psi]$, where $\Psi$ is defined in [3.3. Let $\tau \in K K^{1}(S A, B)$ be represented by $\left[h_{1}\right]-\left[h_{2}\right]$; then $[\tau] \times[\Psi]=0$, by 3.4 Therefore $\left(\left[h_{1}\right]-\left[h_{2}\right]\right) \times[\Psi]=0$, or $\Psi_{*}([\tau])=0$. Hence $\Psi_{*}\left(\left[h_{1}\right]\right)=\Psi_{*}\left(\left[h_{2}\right]\right)$, i.e.,

$$
\left[\pi_{1} \circ H_{1}\right]=\left[\pi_{1} \circ H_{2}\right] \text { in } K K\left(A, \prod_{n=1}^{\infty} B / \bigoplus_{n=1}^{\infty} B\right) \text {. }
$$

Definition 3.6. Let $A$ and $B$ be $C^{*}$-algebras and let $\phi: A \rightarrow B$ be a linear map. We say that $\phi$ is full if the closed ideal generated by $\phi(a)$ is $B$ for each non-zero $a \in A$.

The following was first proved in Ln2. We will give an improvement in 3.9, See also the Remark 3.8 below.

Theorem 3.7. Let $A$ be a separable unital nuclear $C^{*}$-algebra, and let $B$ be a unital $C^{*}$-algebra. Suppose that $h_{1}, h_{2}: A \rightarrow B$ are two unital homomorphisms such that

$$
\left[h_{1}\right]=\left[h_{2}\right] \text { in } K K(A, B) \text {. }
$$

Suppose that $h_{0}: A \rightarrow B$ is a full unital monomorphism. Then, for any $\varepsilon>0$ and finite subset $\mathcal{F} \subset A$, there is an integer $n$ and a unitary $w \in U\left(M_{n+1}(B)\right)$ such that

$$
\left\|w^{*} \operatorname{diag}\left(h_{1}(a), h_{0}(a), \ldots, h_{0}(a)\right) w-\operatorname{diag}\left(h_{2}(a), h_{0}(a), \ldots, h_{0}(a)\right)\right\|<\varepsilon
$$

for all $a \in \mathcal{F}$.

Remark 3.8. The version of Theorem 3.7] in [Ln2 assumed that $A$ is a unital simple $C^{*}$-algebra, and $h_{0}$ is a unital. What one needs is that $\left(h_{0}, h_{0}, \ldots, h_{0}, \ldots\right)$ gives an absorbing extension. From the proof in $\underline{\operatorname{Ln} 2}$, it is easy to see that one only needs to assume that $\phi_{0}(a)$ is full. This was observed in [DE1]. In fact, in [DE1], the nuclearity of $A$ can be replaced by the condition that homomorphisms are nuclear. A simplified and more elementary proof of 3.7 can be found in 5.6.4 in [Ln7]. 
A version of the following was obtained in [Ln2] (see also 5.9.9 in [Ln7]). The following theorem is much more general.

Theorem 3.9. Let $A$ be a separable unital nuclear $C^{*}$-algebra, and let $B$ be a unital $C^{*}$-algebra. Suppose that $h_{1}, h_{2}: A \rightarrow B$ are two unital homomorphisms such that

$$
\left[h_{1}\right]=\left[h_{2}\right] \text { in } K L(A, B) \text {. }
$$

Suppose that $h_{0}: A \rightarrow B$ is a full unital monomorphism. Then, for any $\varepsilon>0$ and finite subset $\mathcal{F} \subset A$, there is an integer $n$ and a unitary $W \in U\left(M_{n+1}(B)\right)$ such that

$$
\left\|W^{*} \operatorname{diag}\left(h_{1}(a), h_{0}(a), \ldots, h_{0}(a)\right) W-\operatorname{diag}\left(h_{2}(a), h_{0}(a), \ldots, h_{0}(a)\right)\right\|<\varepsilon
$$

for all $a \in \mathcal{F}$.

Proof. Let $C=\prod_{n=1}^{\infty} B$, let $C_{0}=\bigoplus_{n=1}^{\infty} B$ and let $\pi: C \rightarrow C / C_{0}$ be the quotient map. Let $H_{1}$ and $H_{2}$ be as in 3.5 Then by [3.5. [ $\left[\pi \circ H_{1}\right]=\left[\pi \circ H_{2}\right]$ in $K K\left(A, \prod_{n=1}^{\infty} B / \bigoplus_{n=1}^{\infty} B\right)$. Let $H_{0}: A \rightarrow \prod_{n=1}^{\infty} B$ be defined by $H_{0}(a)=\left\{h_{0}(a)\right\}$ for all $a \in A$. Since $h_{0}$ is full, it follows that, for any $a \neq 0$, there are $x_{1}, \ldots, x_{n}$ and $y_{1}, \ldots, y_{n} \in B$ such that $\sum_{i=1}^{n} x_{i} h_{0}(a) y_{i}=1_{B}$. Let $X_{i}=\left(x_{i}, x_{i}, \ldots, x_{i}, \ldots\right)$ and $Y_{i}=\left(y_{i}, y_{i}, \ldots, y_{i}, \ldots\right)$ in $\prod_{n=1}^{\infty} B$. Then

$$
\sum_{i=1}^{n} X_{i} H_{0}(a) Y_{i}=1_{C}
$$

This implies that $H_{0}$ is full (in $\prod_{n=1}^{\infty} B$ ). Thus $\pi \circ H_{0}$ is also full. It follows from 3.7. for any $\varepsilon>0$ and any finite subset $\mathcal{F} \subset A$, there is an integer $n$ and a unitary $w \in U\left(M_{n+1}\left(C / C_{0}\right)\right)$ such that

$$
\begin{aligned}
& \| w^{*} \operatorname{diag}\left(\pi \circ H_{1}(a), \pi \circ H_{0}(a), \ldots, \pi \circ H_{0}(a)\right) w \\
& \quad-\operatorname{diag}\left(\pi \circ H_{2}(a), \pi \circ H_{0}(a), \ldots, \pi \circ H_{0}(a)\right) \|<\varepsilon / 2
\end{aligned}
$$

for all $a \in \mathcal{F}$. It follows that there is a unitary $z=\left(W_{1}, W_{2}, \ldots, W_{N}, \ldots\right) \in M_{n+1}(C)$ such that $\pi(z)=w$. Therefore, for sufficiently large $N$,

$$
\left\|W_{N}^{*} \operatorname{diag}\left(h_{1}(a), h_{0}(a), \ldots, h_{0}(a)\right) W_{N}-\operatorname{diag}\left(h_{2}(a), h_{0}(a), \ldots, h_{0}(a)\right)\right\|<\varepsilon
$$

for all $a \in \mathcal{F}$.

Definition 3.10. Denote by $\mathcal{C}_{1}$ the class of $\sigma$-unital $C^{*}$-algebras $A$ satisfying the following:

(1) The canonical homomorphism $U(p A p) / U(p A p)_{0} \rightarrow K_{1}(p A p)$ is an isomorphism, for all full projections $p \in A$.

(2) There is $L>0$ such that $\operatorname{cel}(p A p) \leq L$ for all full projections $p \in A$, i.e., for every $u \in U(p A p)_{0}$, there are self-adjoint elements $h_{1}, \cdots, h_{n} \in p A p$ such that $\sum_{i=1}^{n}\left\|h_{i}\right\| \leq L$ and $u=\exp \left(i h_{1}\right) \cdot \exp \left(i h_{2}\right) \cdots \exp \left(i h_{n}\right)$.

(3) If $p$ and $q$ are two full projections in $A \otimes \mathcal{K}$ with $[p]=[q]$ in $K_{0}(A)$, then there is $w \in A \otimes \mathcal{K}$ such that $w^{*} w=p$ and $w w^{*}=q$.

Every purely infinite simple $C^{*}$-algebra, is in $\mathcal{C}_{1}$ ([Cu] and [P1]).

The following is an important result of Rørdam (Theorem 5.1 in [Rr1]):

Lemma 3.11. Let $A$ be in $\mathcal{C}_{1}$. Suppose that $h_{1}, h_{2}: \mathcal{O}_{2} \rightarrow A$ are two homomorphisms with $h_{1}(1)=p$ and $h_{2}(1)=q$. Suppose that both $p$ and $q$ are full. Then, for 
any $\varepsilon>0$ and any finite subset $\mathcal{F} \subset A$, there is $u \in A$ with $u^{*} u=p$ and $u u^{*}=q$ such that

$$
\left\|u^{*} h_{1}(a) u-h_{2}(a)\right\|<\varepsilon \text { for all } a \in \mathcal{F} .
$$

Definition 3.12. Let $A$ and $B$ be two $C^{*}$-algebras. A homomorphism $h: A \rightarrow B$ is said to factor through $\mathcal{O}_{2}$ if there are homomorphisms $\phi_{1}: A \rightarrow \mathcal{O}_{2}$ and $\phi_{2}$ : $\mathcal{O}_{2} \rightarrow B$ such that $h=\phi_{2} \circ \phi_{1}$.

By the above lemma of Rørdam, for a unital $C^{*}$-algebra, $A$, if $B \in \mathcal{C}_{1}$ and if $h_{1}, h_{2}: A \rightarrow B$ are full homomorphisms and both factor through $\mathcal{O}_{2}$, then, for any $\varepsilon>0$ and any finite subset $\mathcal{F} \subset A$, there is $u \in B$ with $u^{*} u=h_{1}(1)$ and $u u^{*}=h_{2}(1)$ such that

$$
\left\|u^{*} h_{1}(a) u-h_{2}(a)\right\|<\varepsilon \text { for } a \in \mathcal{F} .
$$

The following is a result of Kirchberg ([K2]):

Lemma 3.13. Let $A$ be a unital separable nuclear purely infinite simple $C^{*}$-algebra, and let $B$ be a unital $C^{*}$-algebra, in $\mathcal{C}_{1}$. Suppose that $h: A \rightarrow B$ is a unital homomorphism. Then, for any $\varepsilon>0$ and any finite subset $\mathcal{F} \subset A$, there is $u \in$ $M_{2}(B)$ with $u^{*} u=1_{B}$ and $u u^{*}=1 \oplus p$ such that

$$
\left\|u^{*} \operatorname{diag}\left(h(a), h_{0}(a)\right) u-h(a)\right\|<\varepsilon \text { for all } a \in \mathcal{F}
$$

where $h_{0}: A \rightarrow p B p$ is a unital homomorphism which factors through $\mathcal{O}_{2}$ and $p \in B$ is a full projection. Moreover, $h_{0}$ can be chosen independent of $\varepsilon$ and $\mathcal{F}$.

The following was proved in [P2] (Theorem 4.1.1) under the condition that $\left[h_{1}\right]=\left[h_{2}\right]$ in $K K(A, B)$ (the conclusion there is slightly stronger). This is perhaps precisely the $K L$ analog of the asymptotic unitary equivalence result in [P2]. Moreover, it is also known that under the assumption that $A$ satisfies the UCT, the following theorem holds (see for example, [Ln2]). However, Theorem 3.14 does not assume that $A$ satisfies the UCT nor does it assume that $\left[h_{1}\right]=\left[h_{2}\right]$ in $K K(A, B)$.

Theorem 3.14. Let $A$ be a separable unital nuclear purely infinite simple $C^{*}$ algebra, and let $h_{i}: A \rightarrow B$ be two full homomorphisms, $i=1,2$, where $B$ is a unital $C^{*}$-algebra, in $\mathcal{C}_{1}$. Suppose that

$$
\left[h_{1}\right]=\left[h_{2}\right] \text { in } K L(A, B) .
$$

Then, for any $\varepsilon>0$ and any finite subset $\mathcal{F} \subset A$, there is $u \in B$ with $u^{*} u=h_{2}(1)$ and $u u^{*}=h_{1}(1)$ such that

$$
\left\|u^{*} h_{1}(a) u-h_{2}(a)\right\|<\varepsilon \text { for all } a \in \mathcal{F} .
$$

Proof. To simplify notation, without loss of generality, we may assume that $h_{1}(1)=$ $h_{2}(1)$. By replacing $h_{1}$ by $u^{*}\left(\operatorname{diag}\left(h_{1} \oplus h_{0}\right)(a)\right) u$, as in 3.13 we obtain a full homomorphism $h_{0}^{\prime}: A \rightarrow B$ which factors through $\mathcal{O}_{2}$ ( $h_{0}^{\prime}$ can be taken to be $\left.u h_{0} u^{*}\right)$. By applying [3.9] we obtain an integer $n$ and a unitary $U \in M_{n+1}(B)$ such that

$$
\left\|U^{*} \operatorname{diag}\left(h_{1}(a), h_{0}^{\prime}(a), \ldots, h_{0}^{\prime}(a)\right) U-\operatorname{diag}\left(h_{2}(a), h_{0}^{\prime}(a), \ldots, h_{0}^{\prime}(a)\right)\right\|<\varepsilon / 3
$$

for all $a \in \mathcal{F}$. Since all full homomorphisms that factor through $\mathcal{O}_{2}$ are approximately unitarily equivalent, by applying 3.13 we obtain $V \in M_{n+1}(B)$ with $V^{*} V=$ $h_{1}(1)$ and $V V^{*}=h_{1}(1) \oplus h_{0}^{\prime}(1) \oplus \cdots \oplus h_{0}^{\prime}(1)$ such that

$$
\left\|V^{*} \operatorname{diag}\left(h_{1}(a), h_{0}^{\prime}(a), \ldots, h_{0}^{\prime}(a)\right) V-h_{1}(a)\right\|<\varepsilon / 3
$$


for all $a \in \mathcal{F}$. Similarly, there exists $W \in M_{n+1}(B)$ with $W^{*} W=h_{2}(1)$ and $W W^{*}=h_{2}(1) \oplus h_{0}^{\prime}(1) \oplus \cdots \oplus h_{0}^{\prime}(1)$ such that

$$
\left\|W^{*} \operatorname{diag}\left(h_{2}(a), h_{0}^{\prime}(a), \ldots, h_{0}^{\prime}(a)\right) W-h_{2}(a)\right\|<\varepsilon / 3
$$

for all $a \in \mathcal{F}$. Put $u=V^{*} U W$. Then $u^{*} u=h_{2}(1)$ and $u u^{*}=h_{1}(1)$ such that

$$
\left\|u^{*} h_{1}(a) u-h_{2}(a)\right\|<\varepsilon / 3+\varepsilon / 3+\varepsilon / 3=\varepsilon
$$

for all $a \in \mathcal{F}$.

Remark 3.15. One could add an additional condition that there is a full embedding

from $\mathcal{O}_{2}$ into $C^{*}$-algebras in the class $\mathcal{C}_{1}$. However, in Theorem 3.14, by assuming that there are full homomorphisms from purely infinite simple $C^{*}$-algebras to $B$, we actually assumed that there is a full embedding of $\mathcal{O}_{2}$ to $B$.

\section{The Definition of AUCT}

In this section, we will discuss the definition of the AUCT.

Definition 4.1. Let

$$
0 \rightarrow G_{0} \rightarrow G \stackrel{p}{\rightarrow} G_{1} \rightarrow 0
$$

be a short exact sequence of abelian groups. So $G$ is an extension of $G_{1}$ by $G_{0}$. Recall that the extension is said to be pure if for any finitely generated subgroup $G_{1}^{\prime}$ of $G_{1}$, there is a homomorphism $j: G_{1}^{\prime} \rightarrow G$ such that $p \circ j=\mathrm{id}_{G_{1}^{\prime}}$. The set of pure extensions is denoted by $\operatorname{Pext}\left(G_{1}, G_{0}\right)$. It is a subgroup of $\operatorname{Ext}_{\mathbb{Z}}\left(G_{1}, G_{0}\right)$. We denote by $E_{0}\left(G_{1}, G_{0}\right)$ the quotient $\operatorname{Ext}_{\mathbb{Z}}\left(G_{1}, G_{0}\right) / \operatorname{Pext}\left(G_{1}, G_{0}\right)$. The quotient map will be denoted by $\tilde{\Pi}$.

Definition 4.2. Let $A$ be a nuclear $C^{*}$-algebra, and let $B$ be a $\sigma$-unital $C^{*}$ algebra. Denote by $\gamma_{A}$ (or $\gamma$ if $A$ is understood) the natural map from $\operatorname{Ext}(A, B)$ to $\operatorname{Hom}\left(K_{*}(A), K_{*}(B)\right)$. It was first observed by Larry Brown that there is a homomorphism $\kappa: \operatorname{ker} \gamma \rightarrow \operatorname{Ext}_{\mathbb{Z}}\left(K_{*}(A), K_{*}(B)\right)$. To be more specific, in the case of extensions, if we have a short exact sequence

$$
0 \rightarrow B \rightarrow E \rightarrow A \rightarrow 0,
$$

then the corresponding six-term exact sequence in $K$-theory is

$$
\begin{aligned}
& K_{0}(B) \rightarrow K_{0}(E) \rightarrow K_{0}(A) \\
& \uparrow_{\gamma} \quad \downarrow_{\gamma} \\
& K_{1}(A) \leftarrow K_{1}(E) \leftarrow K_{1}(B) .
\end{aligned}
$$

Thus we have $\gamma: \operatorname{Ext}(A, B) \rightarrow \operatorname{Hom}\left(K_{i}(A), K_{i-1}(B)\right)$. If $\gamma=0$, then the six-term exact sequence breaks into two short exact sequences:

$0 \rightarrow K_{0}(B) \rightarrow K_{0}(E) \rightarrow K_{0}(A) \rightarrow 0 \quad$ and $\quad 0 \rightarrow K_{1}(B) \rightarrow K_{1}(E) \rightarrow K_{1}(A) \rightarrow 0$.

This gives an element in $\operatorname{Ext}_{\mathbb{Z}}\left(K_{i}(A), K_{i}(B)\right)$.

If $x \in \mathcal{T}(A, B)$, then it is straightforward to prove that $\gamma_{A}(x)=0$. So $\mathcal{T}(A, B) \subset$ $\operatorname{ker} \gamma$.

The following is certainly known in the case that $A$ satisfies the Universal Coefficient Theorem. But we do not assume that $A$ satisfies the UCT below.

Lemma 4.3. Let $A$ be a nuclear $C^{*}$-algebra, and let $B$ be a $\sigma$-unital $C^{*}$-algebra. Then

$$
\kappa(\mathcal{T}(A, B)) \subset \operatorname{Pext}\left(K_{*}(A), K_{*}(B)\right)
$$


Proof. We now recall 3.1 and 3.3 and use the notation there. We may assume that $B$ is stable. Let $\tau: A \rightarrow M(B) / B$ be an absorbing approximately trivial extension. By 4.2, $\kappa(\tau)$ gives an element in $\operatorname{Ext}_{\mathbb{Z}}\left(K_{*}(A), K_{*}(B)\right)$ :

$$
0 \rightarrow K_{i}(B) \rightarrow G_{i} \rightarrow K_{i}(A) \rightarrow 0 .
$$

Let $F_{i}=\prod_{n=1}^{\infty} G_{i}$ and let $j: G_{i} \rightarrow F_{i}$ be defined by $j(g)=(g, g, \ldots, g, \ldots)$ (the constant sequence) for $i=0,1$. Let $N_{i}^{(0)}=\bigoplus_{n=1}^{\infty} K_{i}(B)$ and let $\eta_{i}: F_{i} \rightarrow F_{i} / N_{i}^{(0)}$ be the quotient map, $i=0,1$. Note that $K_{i}\left(C_{0}\right)=N_{i}^{(0)}$. It follows from 3.4 that $\Psi_{*}([\tau])=0$. By 2.9 in GL1], since $B$ is stable, $K_{i}(C)=\prod_{n=1}^{\infty} K_{i}(B)$ and $K_{i}\left(C / C_{0}\right)=\prod_{n=1}^{\infty} K_{i}(B) / N_{i}^{(0)}$. Therefore

$$
0 \rightarrow \prod_{n=1}^{\infty} K_{i}(B) / N_{i}^{(0)} \rightarrow \eta_{i} \circ j\left(G_{i}\right) \rightarrow K_{i}(A) \rightarrow 0
$$

splits for $i=0,1$. Let $g \in K_{i}(A)$ such that $k \cdot g=0$ for some positive integer $k$. Suppose that $f \in j\left(G_{i}\right)$ such that $\eta_{i}(f)=g$. Then $k \cdot f \in N_{i}^{(0)}$. Since $f$ is a constant sequence, this implies that $k \cdot f=0$. Therefore the extension

$$
0 \rightarrow K_{i}(B) \rightarrow G_{i} \rightarrow K_{i}(A) \rightarrow 0
$$

is pure.

Definition 4.4. Let $A$ be a nuclear $C^{*}$-algebra, and let $B$ be a $\sigma$-unital $C^{*}$-algebra. Since $\mathcal{T}(A, S B) \subset \operatorname{ker} \gamma_{A}$, we obtain a homomorphism

$$
\bar{\gamma}_{A}: K L(A, B) \rightarrow \operatorname{Hom}\left(K_{*}(A), K_{*}(B)\right) .
$$

It follows from above that $\kappa$ induces a homomorphism

$$
\bar{\kappa}: \operatorname{ker} \bar{\gamma}_{A} \rightarrow E_{0}\left(K_{*}(A), K_{*}(B)\right) .
$$

Definition 4.5. Let $C_{n}$ be a commutative $C^{*}$-algebra, with $K_{0}\left(C_{n}\right)=\mathbb{Z} / n \mathbb{Z}$ and let $K_{1}\left(C_{n}\right)=0$. Suppose that $A$ is a $C^{*}$-algebra. Then $K_{i}(A, \mathbb{Z} / k \mathbb{Z})=K_{i}\left(A \otimes C_{k}\right)$ (see [S2]). One has the following six-term exact sequence (see [S2]):

$$
\begin{aligned}
& K_{0}(A) \quad \rightarrow \quad K_{0}(A, \mathbb{Z} / k \mathbb{Z}) \rightarrow K_{1}(A) \\
& \uparrow_{\mathbf{k}} \quad \downarrow_{\mathbf{k}} \\
& K_{0}(A) \leftarrow K_{1}(A, \mathbb{Z} / k \mathbb{Z}) \leftarrow K_{1}(A) .
\end{aligned}
$$

In [DL2], $K_{i}(A, \mathbb{Z} / n \mathbb{Z})$ is identified with $K K^{i}\left(\mathbb{I}_{n}, A\right)$ for $i=0,1$.

As in [DL2], we use the notation

$$
\underline{K}(A)=\bigoplus_{i=0,1, n \in \mathbb{Z}_{+}} K_{i}(A ; \mathbb{Z} / n \mathbb{Z}) .
$$

By $\operatorname{Hom}_{\Lambda}(\underline{K}(A), \underline{K}(B))$ we mean all homomorphisms from $\underline{K}(A)$ to $\underline{K}(B)$ which respect the direct sum decomposition and the so-called Bockstein operations (see [DL2]). It follows from the definition in [DL2], that if $x \in K K(A, B)$, then the Kasparov product $K K^{i}\left(\mathbb{I}_{n}, A\right) \times x$ gives an element in $K K^{i}\left(\mathbb{I}_{n}, B\right)$ which we identify with $\operatorname{Hom}\left(K_{i}(A, \mathbb{Z} / n \mathbb{Z}), K_{i}(B, \mathbb{Z} / n \mathbb{Z})\right)$. Thus one obtains a map $\Gamma: K K(A, B) \rightarrow$ $\operatorname{Hom}_{\Lambda}(\underline{K}(A), \underline{K}(B))$. It is shown by Dadarlat and Loring ([DL2]) that, if $A$ is in $\mathcal{N}$, then, for any $\sigma$-unital $C^{*}$-algebra, $B$, the map $\Gamma$ is surjective and $\operatorname{ker} \Gamma=$ $\operatorname{Pext}\left(K_{*}(A), K_{*}(B)\right)$. 
Without assuming $A$ satisfies the UCT, we have the following:

Lemma 4.6. Let $A$ and $B$ be a $\sigma$-unital $C^{*}$-algebras. Then the map $\Gamma: K K(A, B)$ $\rightarrow \operatorname{Hom}_{\Lambda}(\underline{K}(A), \underline{K}(B))$ maps $\mathcal{T}(S A, B)$ to zero.

Proof. Without loss of generality, we may assume that $A$ is stable. Let $x \in$ $K K\left(\mathbb{I}_{n}, A\right)$. It follows from [DL1], [Lo1] and [Lo2] that $\mathbb{I}_{n}$ is semiprojective and there is a homomorphism $h: \mathbb{I}_{n} \rightarrow A$ such that $[h]=x$ in $K K\left(\mathbb{I}_{n}, A\right)$. Then, in $K K\left(\mathbb{I}_{n}, A\right) \times K K(A, B), x \times y=h^{*}(y)$ for all $y \in K K(A, B)$. If $y \in \mathcal{T}(S A, B)$, then, by 2.3 $h^{*}(y) \in \mathcal{T}\left(S \mathbb{I}_{n}, B\right)$. Since $\mathbb{I}_{n}$ is separable and nuclear, we may write $K K^{1}\left(S \mathbb{I}_{n}, B\right)=\operatorname{Ext}\left(S \mathbb{I}_{n}, B\right)$. However, $S \mathbb{I}_{n}$ satisfies the UCT. Therefore, since $K_{i}\left(\mathbb{I}_{n}\right)$ is finitely generated, by a result of Schochet [S4] (see also 5.9.11 in [Ln7]), we have $K K^{1}\left(S \mathbb{I}_{n}, B\right)=K L\left(\mathbb{I}_{n}, B\right)$-this can be proved directly without using the UCT. In other words, $\mathcal{T}\left(S \mathbb{I}_{n}, B\right)=0$. Therefore $[x \times \tau]=0$. This implies that $K K\left(\mathbb{I}_{n}, A\right) \times y=0$ for any $y \subset \mathcal{T}(S A, B) \subset K K(A, B)$. By the definition of $\Gamma$ (see [DL2]), we conclude that $\left.\Gamma\right|_{\mathcal{T}(S A, B)}=0$.

Corollary 4.7. Let $A$ and $B$ be $\sigma$-unital $C^{*}$-algebras. Then the map $\Gamma$ gives a map from $K L(A, B)$ to $\operatorname{Hom}_{\Lambda}(\underline{K}(A), \underline{K}(B))$.

Definition 4.8. Let $A$ be a $\sigma$-unital $C^{*}$-algebra. We say $A$ satisfies the Approximate Universal Coefficient Theorem I (AUCT1) if for any $\sigma$-unital $C^{*}$-algebra, $B$, the map $\Gamma$ from $K K(A, B)$ to $\operatorname{Hom}_{\Lambda}(\underline{K}(A), \underline{K}(B))$ is surjective and its kernel is $\mathcal{T}(A, B)$. In other words, we have

$$
K L^{0}(A, B)=\operatorname{Hom}_{\Lambda}(\underline{K}(A), \underline{K}(B)) \quad \text { and } \quad K L^{1}(A, B)=\operatorname{Hom}_{\Lambda}(\underline{K}(A), \underline{K}(S B)) .
$$

Now let $A$ be a separable nuclear $C^{*}$-algebra. We say $A$ satisfies the Approximate Universal Coefficient Theorem II (AUCT2) if for any $\sigma$-unital $C^{*}$-algebra, $B$, the map $\bar{\gamma}: K L(A, B) \rightarrow \operatorname{Hom}\left(K_{*}(A), K_{*}(B)\right)$ is surjective and map $\bar{\kappa}: \operatorname{ker} \bar{\gamma} \rightarrow$ $E_{0}\left(K_{*}(A), K_{*}(B)\right)$ is an isomorphism, i.e., there is an exact sequence

$$
0 \rightarrow E_{0}\left(K_{*}(A), K_{*}(B)\right) \rightarrow K L(A, B) \rightarrow \operatorname{Hom}\left(K_{*}(A), K_{*}(B)\right) \rightarrow 0
$$

which is natural in each variable.

We will show that (AUCT1) is equivalent to (AUCT2) if $A$ is assumed to be separable and nuclear.

It follows from the definition in [DL1] that, if $x \in K K(B, A)$, then the Kasparov product $K K\left(\mathbb{I}_{n}, B\right) \times x$ gives a homomorphism $\Gamma(x): \operatorname{Hom}_{\Lambda}(\underline{K}(A), \underline{K}(D)) \rightarrow$ $\operatorname{Hom}_{\Lambda}(\underline{K}(B), \underline{K}(D))$ for any $C^{*}$-algebra, $D$.

Lemma 4.9. Let $A$ and $B$ be two $\sigma$-unital $C^{*}$-algebras. Suppose that $A$ satisfies the AUCT1 and there is $\bar{x} \in K L(B, A)$ such that $\bar{\gamma}(\bar{x}) \in \operatorname{Hom}\left(K_{*}(B), K_{*}(A)\right)$ is an isomorphism. Then $\Gamma(\bar{x}): \operatorname{Hom}_{\Lambda}(\underline{K}(A), \underline{K}(D)) \rightarrow \operatorname{Hom}_{\Lambda}(\underline{K}(B), \underline{K}(D))$ is an isomorphism.

Proof. Since the map from $K K(B, A)$ to $K L(B, A)$ is surjective, we obtain an element $x \in K K(B, A)$ such that its image in $K L(B, A)$ is $\bar{x}$. The Kasparov product by $x$ gives a map

$$
\Gamma(x): \operatorname{Hom}_{\Lambda}(\underline{K}(A), \underline{K}(D)) \rightarrow \operatorname{Hom}_{\Lambda}(\underline{K}(B), \underline{K}(D))
$$

which is defined, for each $n, i,(i=0,1) \phi_{n, i} \in \operatorname{Hom}\left(K_{i}(B, \mathbb{Z} / n \mathbb{Z}), K_{i}(D, \mathbb{Z} / n \mathbb{Z})\right)$, by

$$
\Gamma(x)\left(\phi_{n, i}\right)(y)=\phi_{n, i}(y \times x),
$$


where $y \in K_{i}(B, \mathbb{Z} / n \mathbb{Z})=K K\left(\mathbb{I}_{n}, B\right)$. It follows that $\Gamma(z)$ gives (for each $k$ ) the following commutative diagram:

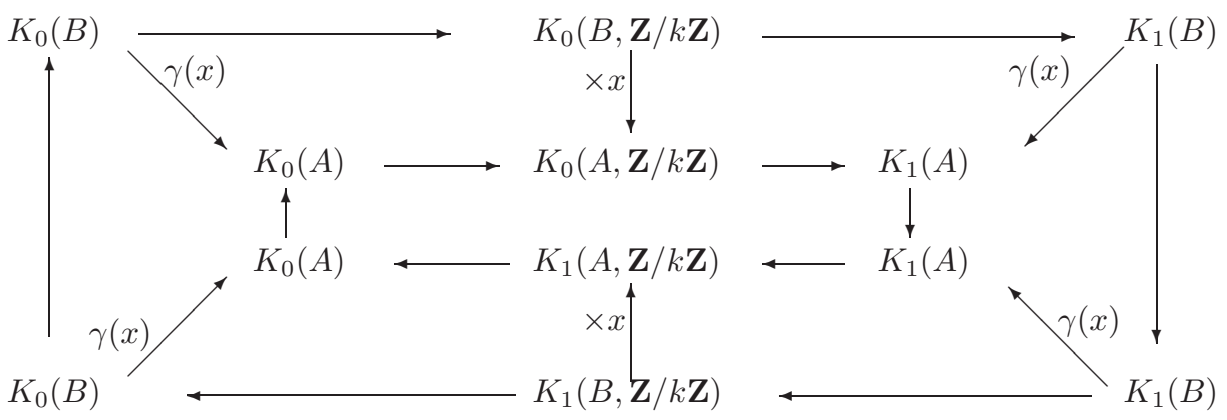

Since $\gamma(x)$ is an isomorphism, by the five lemma, we see that the homomorphism $(-) \times x$ is in fact an isomorphism. By the naturality of the Kasparov product, this implies that $\Gamma(x)$ is an isomorphism.

Lemma 4.10. Let $A$ and $B$ be two nuclear $C^{*}$-algebras and let $D$ be a $\sigma$-unital $C^{*}$-algebra. Suppose that there is an element $x \in K K(A, B)$ such that $\gamma(x)$ is an isomorphism in $\operatorname{Hom}\left(K_{*}(A), K_{*}(B)\right)$. Denote again

$$
\gamma_{A}: K K(A, D) \rightarrow \operatorname{Hom}\left(K_{*}(A), K_{*}(D)\right)
$$

and

$$
\gamma_{B}: K K(B, D) \rightarrow \operatorname{Hom}\left(K_{*}(B), K_{*}(D)\right) .
$$

Then the Kasparov product gives the following (with one arrow missing) commutative diagram:

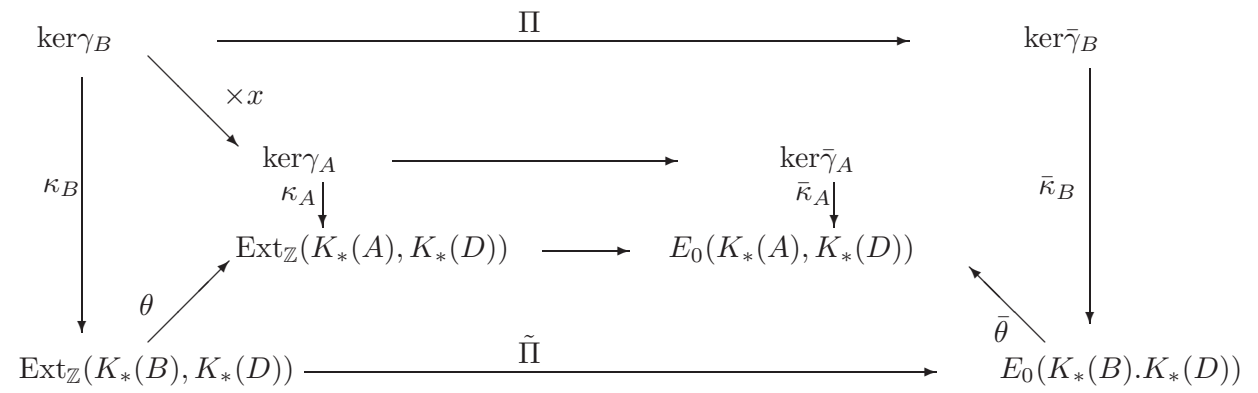

Moreover, both $\theta$ and $\bar{\theta}$ are isomorphisms.

Proof. Fix a geometric injective resolution

$$
0 \rightarrow F \stackrel{g}{\longrightarrow} C \rightarrow S D \rightarrow 0 .
$$

The associated $K$-theory sequence degenerates to

$$
0 \rightarrow K_{i}(D) \rightarrow K_{i}(F) \stackrel{g_{*}}{\longrightarrow} K_{i}(C) \rightarrow 0 \quad(i=0,1)
$$

which is an injective resolution of $K_{i}(D)(i=0,1)$. The six-term exact sequence of $K K$-theory (for any nuclear $C^{*}$-algebra, $A$ ) is

$$
\cdots \rightarrow K K^{i}(A, F) \stackrel{\omega}{\rightarrow} K K^{i}(A, C) \rightarrow K K^{i}(A, D) \rightarrow K K^{i-1}(A, F) \rightarrow \cdots
$$

which unsplices to two exact sequences:

$$
0 \rightarrow \operatorname{coker} \omega \rightarrow K K^{i}(A, D) \rightarrow \operatorname{ker} \omega \rightarrow 0 .
$$


The Kasparov product gives the following commutative diagram:

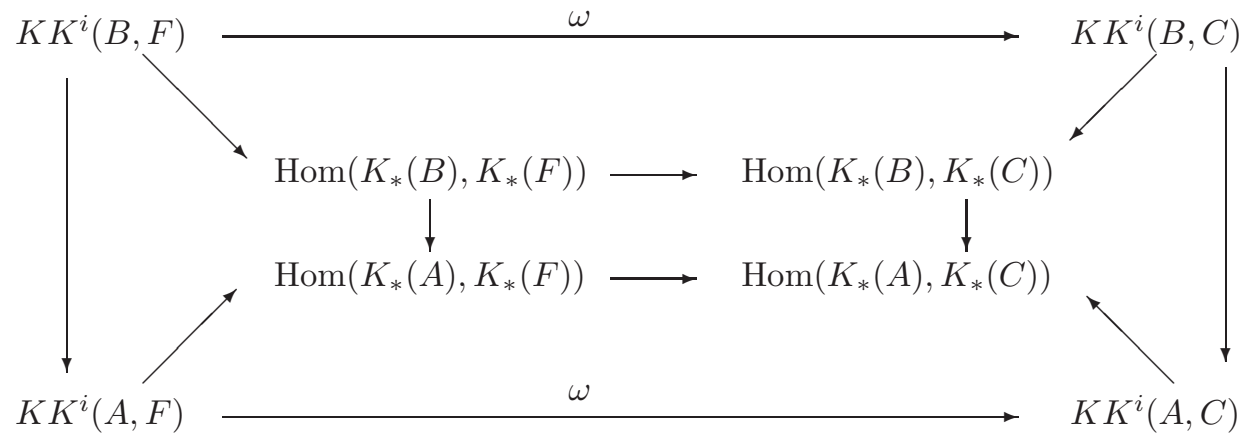

where, the unnamed horizontal maps are $\operatorname{Hom}\left(1, g_{*}\right)$. We identify (see the proof of Theorem 4.1 in $\mathrm{RS}$ )

$$
\operatorname{ker}\left(\operatorname{Hom}\left(1, g_{*}\right)\right)=\operatorname{Hom}\left(K_{*}(A), K_{*}(D)\right)
$$

$\left(\operatorname{ker}\left(\operatorname{Hom}\left(1, g_{*}\right)\right)=\operatorname{Hom}\left(K_{*}(B), K_{*}(D)\right)\right)$ and

$$
\operatorname{coker}\left(\operatorname{Hom}\left(1, g_{*}\right)\right)=\operatorname{Ext}_{\mathbb{Z}}\left(K_{*}(A), K_{*}(D)\right)
$$

$\left(\operatorname{coker}\left(\operatorname{Hom}\left(1, g_{*}\right)\right)=\operatorname{Ext}_{\mathbb{Z}}\left(K_{*}(B), K_{*}(D)\right)\right)$. Since in the above diagram, the two shorter vertical maps are isomorphism, we obtain an isomorphism

$$
\theta: \operatorname{Ext}_{\mathbb{Z}}\left(K_{*}(B), K_{*}(D)\right) \rightarrow \operatorname{Ext}_{\mathbb{Z}}\left(K_{*}(A), K_{*}(D)\right) .
$$

Since $\gamma(x)$ is an isomorphism, $\theta$ maps pure extensions to pure extensions so we also obtain another map

$$
\bar{\theta}: E_{0}\left(K_{*}(B), K_{*}(D)\right) \rightarrow E_{0}\left(K_{*}(A), K_{*}(D)\right) .
$$

Note both $\theta$ and $\bar{\theta}$ are isomorphisms.

Theorem 4.11. Let $A$ be a separable nuclear $C^{*}$-algebra. Then (AUCT1) and (AUCT2) are equivalent.

Proof. (1) AUCT1 implies AUCT2: Suppose that $A$ is a separable nuclear $C^{*}$ algebra, which satisfies the (AUCT1). Let $B \in \mathcal{N}$ such that $K_{i}(A) \cong K_{i}(B)$. Since $A$ satisfies the AUCT1, there is an element $x \in K L^{i}(A, B)$ such that $\bar{\gamma}(x) \in$ $\operatorname{Hom}\left(K_{*}(A), K_{*}(B)\right)$ is an isomorphism. Thus by 4.9 the map $z$ gives an isomorphism $\Pi(z): K L^{i}(A, D) \rightarrow K L^{i}(B, D)$. Let

$$
\bar{\gamma}_{A}: K L(A, D) \rightarrow \operatorname{Hom}\left(K_{*}(A), K_{*}(D)\right)
$$

and

$$
\bar{\gamma}_{B}: K L(B, D) \rightarrow \operatorname{Hom}\left(K_{*}(B), K_{*}(D)\right)
$$

be maps as defined in 4.4. Then the induced maps $\eta: \operatorname{Hom}\left(K_{*}(A), K_{*}(D)\right) \rightarrow$ $\operatorname{Hom}\left(K_{*}(B), K_{*}(D)\right)$ and $\theta: \operatorname{ker} \gamma_{A} \rightarrow \operatorname{ker} \gamma_{B}$ are isomorphisms. On the other hand, by the AUCT1, $\operatorname{ker} \bar{\gamma}_{A}=E_{0}\left(K_{*}(A), K_{*}(D)\right)$. Thus we have the following commutative diagram:

$$
\begin{aligned}
& 0 \rightarrow \quad E_{0}\left(K_{*}(B), K_{*}(D)\right) \rightarrow K L(B, D) \rightarrow \operatorname{Hom}\left(K_{*}(B), K_{*}(D)\right) \quad \rightarrow 0
\end{aligned}
$$

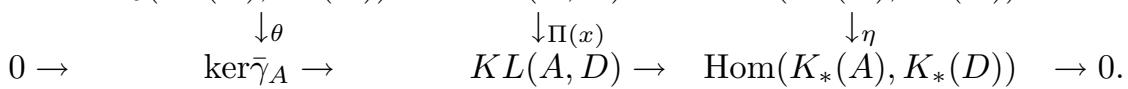


By applying 4.10 we obtain a (complete) commutative diagram:

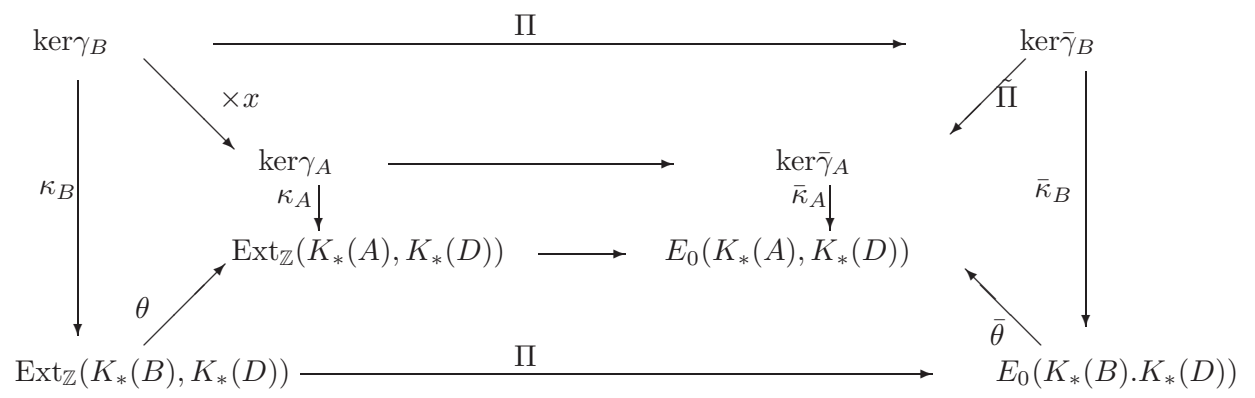

with $\theta$ and $\bar{\theta}$ being isomorphisms. Since $B$ satisfies the UCT, $\bar{\kappa}_{B}$ is an isomorphism. We have also shown that $\Pi(x)$ is an isomorphism. Therefore $\bar{\kappa}_{A}$ is also an isomorphism. This proves that $A$ satisfies (AUCT2).

(2) AUCT2 implies AUCT1: Now we assume that $A$ satisfies the (AUCT2). Again let $B \in \mathcal{N}$ such that $K_{i}(A)=K_{i}(B)$. Since $A$ satisfies the AUCT2, there is an element $x \in K K(A, B)$ such that $\gamma(x)$ gives the isomorphisms. Since $B$ satisfies the UCT, the Kasparov product gives, for any $\sigma$-unital $C^{*}$-algebra, $D$, the following commutative diagram:

$$
\begin{array}{ccccc}
0 \rightarrow & \operatorname{Ext}_{\mathbb{Z}}\left(K_{*}(B), K_{*-1}(D)\right) \rightarrow & K K(B, D) \rightarrow & \operatorname{Hom}\left(K_{*}(B), K_{*}(D)\right) & \rightarrow 0 \\
& \downarrow_{\theta^{\prime}} & \downarrow_{\Pi(x \times(-))} & \downarrow_{\gamma(x)} & \\
0 \rightarrow & E_{0}\left(K_{*}(A), K_{*}(D)\right) \rightarrow & K L(A, D) \rightarrow & \operatorname{Hom}\left(K_{*}(A), K_{*}(D)\right) & \rightarrow 0 .
\end{array}
$$

Applying the commutative diagram of 4.10 , we see that $\theta^{\prime}=\tilde{\Pi} \circ \theta$ (here $\tilde{\Pi}$ is the map from $\operatorname{Ext}_{\mathbb{Z}}\left(K_{*}(A), K_{*}(B)\right)$ to $\left.E_{0}\left(K_{*}(A), K_{*}(D)\right)\right)$. Therefore

$$
\operatorname{ker} \Pi((-) \times x)=\operatorname{Pext}\left(K_{*}(B), K_{*}(D)\right) .
$$

But $\operatorname{Pext}\left(K_{*}(B), K_{*}(D)\right)=\operatorname{Pext}\left(K_{*}(B), K_{*-1}(S D)\right)$ corresponds to $\mathcal{T}(B, S D)$ (see [S4] and 5.9.11 in [Ln7]). Therefore $\operatorname{ker} \Pi((-) \times x)=\mathcal{T}(B, S D)$. So the Kasparov product gives an isomorphism from $K L(B, D)$ to $K L(A, D)$.

On the other hand, $\Gamma(x)$ gives the following commutative diagram (by 4.6):

$$
\begin{array}{llc}
K L(B, D) & \stackrel{\Gamma}{\rightarrow} & \operatorname{Hom}_{\Lambda}(\underline{K}(B), \underline{K}(D)) \\
\downarrow_{\Pi(x \times(-))} & & \downarrow \Gamma(x) \\
K L(A, D) & \stackrel{\Gamma}{\rightarrow} & \operatorname{Hom}_{\Lambda}(\underline{K}(A), \underline{K}(D)) .
\end{array}
$$

From what we have shown the left vertical map is an isomorphism. That the right vertical map is an isomorphism follows from 4.9. Since $B$ satisfies the UCT, it follows from [DL2] the upper horizontal map is also an isomorphism. Thus the above commutative diagram shows that the lower horizontal map has to be surjective and injective. Therefore $A$ satisfies the (AUCT1).

Definition 4.12. Let $A$ be a separable nuclear $C^{*}$-algebra. We say $A$ satisfies the Approximate Universal Coefficient Theorem (AUCT), if either $A$ satisfies the AUCT1 or AUCT2.

Corollary 4.13. Suppose that $A$ and $B$ are two $\sigma$-unital nuclear $C^{*}$-algebras which satisfy the AUCT. Suppose that $K_{i}(A) \cong K_{i}(B)(i=0,1)$. Then there are elements $z \in K L(A, B)$ and $z^{-1} \in K L(B, A)$ such that $\Gamma\left(z^{-1}\right)$ is an isomorphism from $\operatorname{Hom}_{\Lambda}(\underline{K}(A), \underline{K}(D))$ onto $\operatorname{Hom}_{\Lambda}(\underline{K}(B), \underline{K}(D))$ for any $\sigma$-unital $C^{*}$-algebra, $D$ with inverse given by $\Gamma(z)$. 
Theorem 4.14. Let $A$ be a separable nuclear $C^{*}$-algebra, which satisfies the AUCT and let $D$ be a $\sigma$-unital $C^{*}$-algebra. If $\tau \in \operatorname{Ext}(A, D)$ so that $\tau$ gives two pure extensions

$$
0 \rightarrow K_{i}(D) \rightarrow K_{i}(E) \rightarrow K_{i}(A) \rightarrow 0,
$$

then $\tau$ is stably approximately trivial.

Proof. Suppose that $[\tau] \in \operatorname{Ext}(A, D)=K K(A, D)$ so that $\tau$ gives two pure extensions as described in the theorem. By the AUCT (AUCT2), $\Pi([\tau])=0$. So $\tau$ is stably approximately trivial.

\section{Applications to Classification of Simple nuClear $C^{*}$-Algebras}

Definition 5.1. Let $A_{1}, A_{2}, \ldots, A_{n}, \ldots$ and $B_{1}, B_{2}, \ldots, B_{n}, \ldots$ be two sequences of $C^{*}$-algebras. Let $\varepsilon_{n}, \delta_{n}$ and $\eta_{n}$ be decreasing sequences of positive numbers such that

$$
\sum_{n=1}^{\infty} \varepsilon_{n}<\infty, \sum_{n=1}^{\infty} \delta_{n}<\infty \text { and } \sum_{n=1}^{\infty} \eta_{n}<\infty
$$

Let $\phi_{n}: A_{n} \rightarrow A_{n+1}, \psi_{n}: B_{n} \rightarrow B_{n+1}$ and $L_{n}: A_{n} \rightarrow B_{n}$ be sequences of contractive completely positive linear maps. Suppose that $\mathcal{F}_{1}^{(n)} \subset \mathcal{F}_{2}^{(n)} \subset \cdots \subset$ $\mathcal{F}_{k}^{(n)} \subset \cdots$ and $\mathcal{G}_{1}^{(n)} \subset \mathcal{G}_{2}^{(n)} \subset \cdots \subset \mathcal{G}_{k}^{(n)} \subset \cdots$ be finite subsets of $A_{n}$ and $B_{n}$ such that $\bigcup_{k} \mathcal{F}_{k}^{(n)}$ and $\bigcup_{k} \mathcal{G}_{k}^{(n)}$ are dense in $A_{n}$ and $B_{n}$, respectively $(n=1,2, \ldots)$. Suppose also that $\phi_{n} \circ \cdots \circ \phi_{k}\left(\mathcal{F}_{k}^{(n)}\right) \subset \mathcal{F}_{1}^{(n+1)}$ and $\psi_{n} \circ \cdots \circ \psi_{k}\left(\mathcal{G}_{k+1}^{(n)}\right) \subset \mathcal{G}_{1}^{(n+1)}$. Moreover

$$
\left\|\phi_{n}\left(a^{\prime} a\right)-\phi_{n}\left(a^{\prime}\right) \phi_{n}(a)\right\|<\varepsilon_{n}, \quad\left\|L_{n}\left(a^{\prime} a\right)-L_{n}\left(a^{\prime}\right) L_{n}(a)\right\|<\delta_{n}
$$

for all $a, a^{\prime} \in \mathcal{F}_{1}^{(n)}$, and

$$
\left\|\psi_{n}\left(b b^{\prime}\right)-\psi_{n}(b) \psi_{n}\left(b^{\prime}\right)\right\|<\eta_{n}
$$

for all $b, b^{\prime} \in \mathcal{G}_{1}^{(n)}$. Set $A=\lim _{n}\left(A_{n}, \phi_{n}\right)$ and $B=\lim _{n}\left(B_{n}, \psi_{n}\right)$ (generalized inductive limits in the sense in BK1]). Suppose further that

$$
\left\|\psi_{n+1} \circ L_{n}(a)-L_{n+1} \circ \phi_{n}(a)\right\|<\varepsilon_{n}
$$

for all $a \in \mathcal{F}_{1}^{(n)}$. Then we say the following diagram is (one-sided) approximately intertwining:

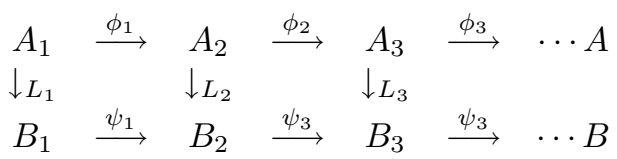

It follows from an argument of Elliott that there is a homomorphism $h: A \rightarrow B$ which completes the above approximated intertwining diagram (see [Ell1] and for a more elaborated late version, see 1.10.14 and 1.10.15 [Ln7]).

If furthermore, there are contractive completely positive linear map $H_{n}: B_{n} \rightarrow$ $A_{n+1}$ such that

$$
\left\|H_{n}\left(b b^{\prime}\right)-H_{n}(b) H_{n}\left(b^{\prime}\right)\right\|<\delta_{n},\left\|H_{n} \circ L_{n}(a)-\phi_{n}(a)\right\|<\eta_{n}
$$

and

$$
\left\|L_{n+1} \circ H_{n}(b)-\psi_{n}(b)\right\|<\eta_{n}
$$

for all $b, b^{\prime} \in \mathcal{G}_{1}^{(n)}$ and $a \in \mathcal{F}_{1}^{(n)}$, then there are isomorphisms $h: A \rightarrow B$ and $h^{-1}: B \rightarrow A$. 
Definition 5.2. Let $\phi_{i}: A \rightarrow B$ be two maps $(i=1,2)$ and $\mathcal{F} \subset A$. For $\varepsilon>0$, we will write

$$
\begin{gathered}
\phi_{1} \approx_{\varepsilon} \phi_{2}, \text { on } \mathcal{F}, \text { if } \\
\left\|\phi_{1}(a)-\phi_{2}(a)\right\|<\varepsilon \text { for all } a \in \mathcal{F} .
\end{gathered}
$$

The following result strengthens (marginally) the Kirchberg and Phillips's ([P2] and [K2]) classification theorem. With 3.14, we can replace the assumption that $A$ and $B$ satisfy the UCT by the condition that they satisfy the AUCT. The possibility of this approach first appeared in [Ln2].

Theorem 5.3. Let $A$ and $B$ be two separable nuclear purely infinite simple $C^{*}$ algebras satisfying the AUCT.

(i) If both $A$ and $B$ are unital, then $A \cong B$ if and only if

$$
\left(K_{0}(A),\left[1_{A}\right]\right) \cong\left(K_{0}(B),\left[1_{B}\right]\right) \text { and } K_{1}(A) \cong K_{1}(B) .
$$

(ii) If both $A$ and $B$ are stable, then $A \cong B$ if and only if

$$
K_{i}(A) \cong K_{i}(B), \quad i=0,1 .
$$

Proof. We will prove (i) only. The "only if" part follows easily. So we will prove the "if" part. Let $\alpha \in \operatorname{Hom}\left(K_{*}(A), K_{*}(B)\right)$ be an isomorphism as given. By 4.13 since both $A$ and $B$ satisfy the AUCT, there are elements $x \in K K(A, B)$ and $y \in K K(B, A)$ such that $\Pi(x \times y)=\left[\operatorname{id}_{A}\right]$ and $\Pi(y \times x)=\left[\operatorname{id}_{B}\right]$ in $K L(A, A)$ and $K L(B, B)$, respectively. It follows by 4.1.1 in [P2] that there are unital homomorphisms $h_{1}: A \rightarrow B$ and $\phi_{1}^{\prime}: B \rightarrow A$ such that $\left[h_{1}\right]=[x]$ and $\left[\phi_{1}^{\prime}\right]=[y]$. Moreover $\left[\phi_{1}^{\prime} \circ h_{1}\right]=\left[\mathrm{id}_{A}\right]$ in $K L(A, A)$ and $\left[h_{1} \circ \phi_{1}^{\prime}\right]=\left[\mathrm{id}_{B}\right]$ in $K L(B, B)$.

Let $\mathcal{F}_{1} \subset \mathcal{F}_{2} \subset \cdots$ be finite subsets such that $\bigcup_{n=1}^{\infty} \mathcal{F}_{n}$ is dense in $A$. Let $\mathcal{G}_{1} \subset \mathcal{G}_{2} \subset \cdots$ be finite subsets such that $\bigcup_{n=1}^{\infty} \mathcal{G}_{n}$ is dense in $B$.

It follows from 3.14 that there is a unitary $u_{1} \in A$ such that

$$
\operatorname{ad} u \circ \phi_{1}^{\prime} \circ h_{1} \approx_{1 / 2} \operatorname{id}_{A} \text { on } \mathcal{F}_{1} \text {. }
$$

Set $\phi_{1}=\operatorname{ad} u \circ \phi_{1}^{\prime}$. Let $S_{1}=\mathcal{F}_{1}$ and $S_{1}^{\prime}=\mathcal{G}_{1} \cup h_{1}\left(S_{1}\right)$. It follows again from 3.14 that there is a unitary $v_{1} \in B$ such that

$$
\operatorname{ad} v \circ h_{1} \circ \phi_{1} \approx_{1 / 4} \operatorname{id}_{B} .
$$

Define $h_{2}: A \rightarrow B$ by ad $v_{1} \circ h_{1}$. If we continue this process, we obtain the following approximately intertwining diagram:

$$
\begin{array}{cccccccc}
A & \stackrel{\operatorname{id}_{A}}{\longrightarrow} & A & \stackrel{\operatorname{id}_{A}}{\longrightarrow} & A & \stackrel{\operatorname{id}_{A}}{\longrightarrow} & \cdots \longrightarrow & A \\
\downarrow_{h_{1}} & \nearrow_{\phi_{1}} & \downarrow_{h_{2}} & \nearrow_{\phi_{2}} & \downarrow_{h_{3}} & & & \\
B & \stackrel{\operatorname{id}_{B}}{\longrightarrow} & B & \stackrel{\operatorname{id}_{B}}{\longrightarrow} & B & \stackrel{\operatorname{id}_{B}}{\longrightarrow} & \cdots \longrightarrow & B .
\end{array}
$$

Therefore $A \cong B$.

In the absence of the AUCT, we have the following version of the KirchbergPhillips theorem:

Theorem 5.4. Let $A$ and $B$ be two separable nuclear purely infinite simple $C^{*}$ algebras.

(i) If both $A$ and $B$ are non-unital and there is $x \in K K(A, B)$ and $y \in K K(B, A)$ such that $\Pi(x \times y)=\left[\operatorname{id}_{A}\right]$ and $\Pi(y \times x)=\left[\operatorname{id}_{B}\right]$, then $A \cong B$.

(ii) If both $A$ and $B$ are unital, and there is $x \in K K(A, B)$ and $y \in K K(B, A)$ such that $\Pi(x \times y)=\left[\operatorname{id}_{A}\right], \Pi(y \times x)=\left[\operatorname{id}_{B}\right]$ and $\gamma(x)\left(\left[1_{A}\right]\right)=\left[1_{B}\right]$, then $A \cong B$. 
Corollary 5.5. Let $A$ be a separable nuclear purely infinite simple $C^{*}$-algebra. If $A$ satisfies the $A U C T$, then $A \in \mathcal{N}$.

Proof. The proof of this follows from the fact that separable nuclear purely infinite simple $C^{*}$-algebras in $\mathcal{N}$ exhaust all possible $K_{i}$-groups $([\mathrm{Rr} 2])$ and the above theorem.

Definition 5.6. Let $A$ be a $C^{*}$-algebra, and let $C_{n}$ be as in Definition 4.5. Let $\mathbf{P}(A)$ be the set of all projections in $M_{\infty}(A), M_{\infty}\left(C\left(S^{1}\right) \otimes A\right), M_{\infty}\left(A \otimes C_{m}\right)$ and $\left.M_{\infty}\left(C\left(S^{1}\right) \otimes A \otimes C_{m}\right)\right)$. Let $B$ be another $C^{*}$-algebra, and let $L: A \rightarrow B$ be a completely positive linear map. Then $L$ induces maps from $A \otimes C_{m} \rightarrow B \otimes C_{m}$, from $C\left(S^{1}\right) \otimes A \otimes C_{m}$ to $C\left(S^{1}\right) \otimes B \otimes C_{m}$, namely, $L \otimes$ id. For convenience, we will also denote the induced map by $L$.

Let $A$ and $B$ be $C^{*}$-algebras, let $L: A \rightarrow B$ be a contractive completely positive linear map, let $\varepsilon>0$ and let $\mathcal{F} \subset A$ be a subset. $L$ is said to be $\mathcal{F}$ - $\varepsilon$-multiplicative if

$$
\|L(x y)-L(x) L(y)\|<\varepsilon
$$

for all $x, y \in \mathcal{F}$.

Given a projection $p \in \mathbf{P}(A)$, if $L$ is $\mathcal{G}$ - $\varepsilon$-multiplicative with sufficiently large $\mathcal{G}$ and sufficiently small $\varepsilon, L(p)$ is close to a projection. Let $L(p)^{\prime}$ be that projection. Fix finite subsets of $\mathcal{P}_{1} \subset \mathbf{P}(A)$. It is easy to see that $L(p)^{\prime}$ and $L(q)^{\prime}$ are in the same equivalence class of projections of $\mathbf{P}(A)$ if $p$ and $q$ are in $\mathcal{P}_{1}$ and are in the same equivalence class of projections of $\mathbf{P}(A)$, provided that $\mathcal{F}$ is sufficiently large and $\varepsilon$ is sufficiently small. We use $[L](p)$ for the class of the projections containing $L(p)^{\prime}$.

In what follows, whenever we write $[L](p)$, we assume that $\mathcal{F}$ is sufficiently large and $\varepsilon$ is sufficiently small so that $[L](p)$ are well defined on $\mathcal{P}_{1}$.

Suppose that $q$ is in $\mathbf{P}(A)$ with $[q]=k[p]$ for some integer $k$, by adding sufficiently many elements (partial isometries) in $\mathcal{F}$, we can assume that $[L](q)=k[L](p)$.

Suppose that $G$ is a finitely generated group generated by $\mathcal{P}$ and $G=\mathbb{Z}^{n} \oplus$ $\mathbb{Z} / k_{1} \mathbb{Z} \oplus \cdots \mathbb{Z} / k_{m} \mathbb{Z}$. Let $g_{1}, g_{2}, \ldots, g_{n}$ be free generators of $\mathbb{Z}^{n}$ and let $t_{i} \in \mathbb{Z} / k_{i} \mathbb{Z}$ be the generator with order $k_{i}, i=1,2, \ldots, m$. Since every element in $K_{0}(C)$ (for any unital $C^{*}$-algebra, $C$ ) may be written as $\left[p_{1}\right]-\left[p_{2}\right]$ for projections $p_{1}, p_{2} \in$ $A \otimes M_{l}$, for some $l>0$, with sufficiently large $\mathcal{F}$ and sufficiently small $\varepsilon$, one can define $[L]\left(g_{j}\right)$ and $[L]\left(t_{i}\right)$. Moreover (with sufficiently large $\mathcal{F}$ and sufficiently small $\varepsilon)$, the order of $[L]\left(t_{i}\right)$ divides $k_{i}$. Then we can define a map $\left.[L]\right|_{G}$ by defining $[L]\left(\sum_{i}^{n} n_{i} g_{i}+\sum_{j}^{m} m_{j} t_{j}\right)=\sum_{i}^{k} n_{i}[L]\left(g_{i}\right)+\sum_{j}^{m} m_{j}[L]\left(t_{j}\right)$. Note, in general, that $\left.[L]\right|_{\mathcal{P}}$ may not coincide with $\left.[L]\right|_{G}$ on $\mathcal{P}$. However, if $\mathcal{F}$ is large enough and $\varepsilon$ is small enough, they coincide. In what follows, we say $\left.[L]\right|_{G}$ is well defined and write $\left.[L]\right|_{G}$ if $k_{i}$,

(1) $[L]$ is well defined on $\left\{g_{1}, g_{2}, \ldots, g_{n}, t_{1}, \ldots, t_{m}\right\}$ with the order of $[L]\left(t_{i}\right)$ dividing

(2) $\left.[L]\right|_{\mathcal{P}}=\left.[L]\right|_{G}$ on $\mathcal{P}$.

(See 1.6 and 1.8 in Ln4.)

Definition 5.7 (4.6 in [Ln2], see also [GL2]). Fix $l \geq 1, b \geq \pi$ and $M \geq 1$. We say a unital $C^{*}$-algebra $A \in \mathbf{C}_{(l, b, M)}$ if

(a) for any projections $p, q \in M_{K}(A)$ with $[p]=[q]$ in $K_{0}(A), p \oplus 1_{M_{K l}(A)}$ is Murry-von Neumann equivalent to $q \oplus 1_{M_{K l}(A)}$ for all $K$,

(b) the canonical map $U\left(M_{l}(A)\right) / U_{0}\left(M_{l}(A)\right) \rightarrow K_{1}(A)$ is surjective, 
(c) the exponential length of $M_{m}(A), \operatorname{cel}\left(M_{m}(A)\right) \leq b$ for all $m$,

(d) if $k>0$ and $-l\left[1_{A}\right] \leq k x \leq l\left[1_{A}\right]$, then $-l M k\left[1_{A}\right] \leq x \leq l M k\left[1_{A}\right]$ for all $x \in K_{0}(A)$.

If $A$ is a separable $C^{*}$-algebra, with real rank zero, stable rank one and with weakly unperforated $K_{0}(A)$, then $A \in \mathbf{C}_{1, \pi, 1}$ (see [GL2]). In particular, if $A$ is a simple $C^{*}$-algebra, with $T R(A)=0$, then $A \in \mathbf{C}_{1, \pi, 1}$ ([Ln6]).

Definition 5.8. A contractive completely positive linear map $L: A \rightarrow B$, where $B$ is unital, is said to be $(N(a), K(a))_{a \in \mathcal{F}}$-full, if for each $a \in \mathcal{F} \subset A_{+} \backslash\{0\}$ there are $x_{1}(a), \ldots, x_{N(a)}(a) \in B$ such that $\left\|x_{i}(a)\right\| \leq K(a)$ and

$$
\sum_{i=1}^{N(a)} x_{i}(a)^{*} L(a) x_{i}(a)=1_{B} .
$$

Also if $\mathcal{F} \subset A$, we denote by $\mathcal{F}^{+}$the set $\left\{\left(\frac{\left(a^{*}+a\right)}{2}\right)_{+},\left(\frac{\left(a-a^{*}\right)}{2 i}\right)_{+}: a \in \mathcal{F}\right\} \backslash\{0\}$.

With 3.9, exactly as in the proof of Theorem 5.3 in [Ln2] (see also 6.3.1 in [Ln7]), we obtain the following:

Theorem 5.9. Let $A$ be a nuclear separable $C^{*}$-algebra, satisfying the AUCT and $l \geq 1, b \geq \pi$ and $M \geq 1$. Then, for any finite subset $\mathcal{F} \subset A, \varepsilon>0$ and $T$ : $A \rightarrow\left(\mathbb{N}_{+}, \mathbb{R}_{+}\right), a \mapsto(N(a), K(a))$, there exist a finite subset $\mathcal{G} \subset A$, a positive number $\delta>0$, a finite subset $\mathcal{P} \subset \mathbf{P}(A)$ (they do not depend on $T$ but depends on $A, \varepsilon$ and $\mathcal{F})$ such that, for any unital $C^{*}$-algebra, $B \in \mathbf{C}_{(l, b, M)}$ and any $\mathcal{G}$ $\delta$-multiplicative contractive completely positive linear maps, $\phi, \psi: A \rightarrow B$ and any $\left\{(N(a), K(a)): a \in \mathcal{G}^{+}\right\}$-full unital $\mathcal{G}$ - $\delta$-multiplicative contractive completely positive linear map $\sigma: A \rightarrow B$, if

$$
\left.\left[\phi_{1}\right]\right|_{\mathcal{P}}=\left.\left[\phi_{2}\right]\right|_{\mathcal{P}},
$$

then there exists an integer $k>0$ and a unitary $U \in M_{k+1}(B)$ such that

$$
\left\|U^{*}(\operatorname{diag}(\phi(a), \sigma(a), \ldots, \sigma(a))) U-\operatorname{diag}(\psi(a), \sigma(a), \ldots, \sigma(a))\right\|<\varepsilon
$$

for all $a \in \mathcal{F}$.

Proof. The proof is merely a minor modification of that of 5.3 in Ln2 (see also 6.3.1 in [Ln7]). We will refer to that proof. The only thing that we are required to add to the proof of 5.3 in [Ln2] (6.3.1 in [Ln]]) is to show that $\bar{\Sigma}: A \rightarrow \prod_{n} B_{n} / \bigoplus_{n} B_{n}$ is a full monomorphism. Given $a \in A_{+} \backslash\{0\}$, there are $x_{i}^{(n)}(a) \in B_{n}, i=1, \ldots, N(a)$, such that $\left\|x_{i}^{(n)}(a)\right\| \leq K(a)$ and

$$
\sum_{i=1}^{N(a)} x_{i}^{(n)}(a)^{*} \sigma_{n}(a) x_{i}^{(n)}(a)=1_{B_{n}}
$$

for all large $n$. Let $x_{i}(a)=\left\{x_{i}^{(n)}\right\}$ (with any first few $x_{i}^{(n)}(a)$ ). Then $x_{i}(a) \in \prod_{n} B_{n}$ such that

$$
\sum_{i=1}^{N(a)} \pi\left(x_{i}(a)\right)^{*} \bar{\Sigma}(a) \pi\left(x_{i}(a)\right)=1_{C / C_{0}}
$$

where $C=\prod_{n} B_{n}, C_{0}=\bigoplus_{n} B_{n}$ and $\pi: C \rightarrow C / C_{0}$ as in the proof of 5.3 in [Ln2]. This shows that $\bar{\Sigma}$ is full as well as injective (since $\bar{\Sigma}(a) \neq 0$ for any $a \in A$ ). The rest of the proofs are exactly the same. 
The following theorem was proved in [Ln8] with the condition that both $A$ and $B$ satisfy the UCT. With [5.9, we can strengthen it as follows.

Theorem 5.10. Let $A$ and $B$ be two separable unital nuclear simple $C^{*}$-algebras with tracial topological rank zero and satisfying the $A U C T$. Then $A \cong B$ if and only if

$$
\left(K_{0}(A), K_{0}(A)_{+},\left[1_{A}\right], K_{1}(A)\right) \cong\left(K_{0}(B), K_{0}(B)_{+},\left[1_{B}\right], K_{1}(B)\right) .
$$

Since there are separable unital simple $C^{*}$-algebras $A$ in $\mathcal{N}$ with $T R(A)=0$ with any given weakly unperforated $K_{0}(A)$ with the Reisz property and countable abelian group $K_{1}(A)$, we obtain the following

Corollary 5.11. Let $A$ be a unital separable nuclear simple $C^{*}$-algebra, with tracial topological rank zero and satisfying the AUCT. Then $A$ is in $\mathcal{N}$.

\section{An approximate Universal Coefficient Theorem}

In this section, we establish a theorem which says a certain class of $C^{*}$-algebras satisfies the approximate Universal Coefficient Theorem for $K L$. By [RS], we note that all $C^{*}$-algebras in $\mathcal{N}$ satisfy the UCT. We will show that $C^{*}$-algebras that are "locally in" $\mathcal{N}$ satisfy the AUCT. Moreover, we show that $C^{*}$-algebras which "locally" satisfy the AUCT satisfy the AUCT. We start with the following lemma.

Lemma 6.1. Suppose that $B$ is a $\sigma$-unital stable $C^{*}$-algebra. Then $Q(B) \in$ $\mathbf{C}_{(1,3 \pi, 1)}$.

Proof. Since $B$ is stable, for any $m>0$, there is a partial isometry $v \in M_{m}(Q(B))$ such that

$$
v^{*} v=1_{Q(B)} \quad \text { and } \quad v v^{*}=1_{M_{m}(Q(B))} .
$$

Thus, every projection in $M_{m}(Q(B))$ is equivalent to a projection in $Q(B)$. This implies that $Q(B)$ satisfies condition (d) in 5.7 for $M=1$. Furthermore, suppose that $p, q \in M_{l}(Q(B))$ and $[p]=[q]$ in $K_{0}(Q(B))$. We may assume that $p \oplus 1_{M_{m}(Q(B))}$ is equivalent to $q \oplus 1_{M_{m}(Q(B))}$. This implies (a) in 5.7

Let $u \in U\left(M_{m}(Q(B))\right.$. Then

$$
v^{*} u v \in U(Q(B)) .
$$

It is standard that $\operatorname{diag}\left(v^{*} u v, 1_{M_{2 m}(Q(B))}\right)$ and $\operatorname{diag}\left(u, 1_{M_{m}(Q(B))}\right)$ are in the same path-connected component of $U\left(M_{2 m+1}(Q(B))\right.$. This proves that $Q(B)$ satisfies (b) for $l=1 \mathrm{in} 5.7$.

Let $u \in U_{0}\left(M_{m}(Q(B))\right.$. There is a unitary $w \in M_{m}(M(B))=M\left(M_{m}(B)\right)$. It follows from [Zh2] (see also [M]) that

$$
\operatorname{cel}\left(M\left(M_{m}(B)\right)\right) \leq 3 \pi .
$$

This implies that $\operatorname{cel}\left(M_{m}(Q(B))\right) \leq 3 \pi($ for all $m)$.

Definition 6.2. Let $A$ be a nuclear and separable $C^{*}$-algebra, and let $h: A \rightarrow$ $B\left(l^{2}\right)$ be an injective homomorphism such that $\pi \circ h: A \rightarrow B\left(l^{2}\right) / \mathcal{K}$ is also injective, where $\pi: B\left(l^{2}\right) \rightarrow B\left(l^{2}\right) / \mathcal{K}$ is the quotient map. Suppose that $B$ is $\sigma$-unital. There is an obvious embedding $\imath: B\left(l^{2}\right) \rightarrow M(B \otimes \mathcal{K})$. The composition $\imath \circ h: A \rightarrow$ $M(B \otimes \mathcal{K})$ gives an absorbing trivial extension $\tau_{0}=\pi \circ \imath \circ h: A \rightarrow Q(B)$ (see [Ka] $)$. 
We have the following:

Proposition 6.3. Let $\tau: A \rightarrow Q(B)$ be an injective homomorphism. If $\tau$ gives an absorbing extension, then $\tau$ is full.

Proof. Let $\tau_{0}=\pi \circ \imath \circ h$ be the trivial absorbing extension defined 6.2. We will show that $\tau_{0}$ is full. Let $a \in A$ be a non-zero element. The embedding $\imath: B\left(l^{2}\right) \rightarrow$ $M(B \otimes \mathcal{K})$ gives an embedding $\bar{\imath}: B\left(l^{2}\right) / \mathcal{K} \rightarrow Q(B)$. Let $D=\bar{\imath}\left(B\left(l^{2}\right) / \mathcal{K}\right)$. Then $D$ is a unital simple $C^{*}$-algebra. Thus the (closed) ideal generated by $\tau_{0}(a)$ contains the identity of $D$. Since the identity of $D$ is the identity of $Q(B)$, we see that $\tau_{0}$ is full. Therefore $\tau_{0} \oplus \tau$ is also full. Since $\tau_{0} \oplus \tau$ is unitarily equivalent to $\tau, \tau$ is full.

Lemma 6.4. Let $A$ be a $C^{*}$-algebra, with real rank zero and let $a \in A_{+} \backslash\{0\}$. Then for any $\varepsilon>0$, there is a non-zero projection $p \in A$ such that

$$
a \geq(\|a\|-\varepsilon) p .
$$

Proof. Let $f \in C_{0}((0,\|a\|])+$ such that $f(t)=0$ if $t \in[0,\|a\|-\varepsilon]$ and $f(t)=1$ if $t \in$ $[\|a\|-\varepsilon / 2,\|a\|]$. Set $b=f(a)$. Then $b \neq 0$. Let $B=\operatorname{Her}(b)=\overline{b A b}$. Since $R R(A)=0$, there exists a non-zero projection $p \in B$. It is clear from the construction that

$$
a \geq(\|a\|-\varepsilon) p .
$$

Lemma 6.5. Let $A$ be any separable $C^{*}$-algebra, and let $B$ be a unital purely infinite simple $C^{*}$-algebra. Let $\mathcal{G}$ be a finite subset of $A_{+}$. Suppose that $\phi: A \rightarrow B$ is a contractive completely positive linear map such that $\|\phi(a)\| \geq 1 / 2\|a\|$ for all $a \in \mathcal{G}$. Suppose that $(N(a), K(a))=(1,3)$ for all $a \in \mathcal{G}$. Then $\phi$ is $(1,3))_{a \in \mathcal{G} \text {-full. }}$

Proof. It follows from [Zh1] that $R R(B)=0$. If follows from Lemma 6.4 that

$$
\phi(a) \geq 1 / 3 p \text { for some nonzero projection in } B \text {. }
$$

Since $B$ is a purely infinite simple $C^{*}$-algebra, there is a projection $e \leq p$ and a partial isometry $v \in B$ such that $v^{*} v=1$ and $v v^{*}=e$. Hence $v^{*} p v=1$. Thus we obtain an element $c \in B$ with $\|c\| \leq 3$ such that

$$
c^{*} \phi(a) c=1 \text {. }
$$

Theorem 6.6. Let $A$ be a nuclear separable $C^{*}$-algebra, satisfying the AUCT. Then, for any finite subset $\mathcal{F} \subset A$ and $\varepsilon>0$ there exist a finite subset $\mathcal{G} \subset A, a$ positive number $\delta>0$ and a finite subset $\mathcal{P} \subset \mathbf{P}(A)$ such that, for any $\sigma$-unital stable $C^{*}$-algebra, $B$ and any $\mathcal{G}$ - $\delta$-multiplicative contractive completely positive linear maps $\phi, \psi: A \rightarrow Q(B)$ and any $\mathcal{G}$ - $\delta$-multiplicative contractive completely positive linear map $\sigma: A \rightarrow Q(B)$ which satisfies $\|\sigma(a)\| \geq 1 / 2\|a\|$ for all $a \in \mathcal{G}^{+}$and $\phi$ factors through $Q(\mathcal{K})$, if

$$
\left.\left[\phi_{1}\right]\right|_{\mathcal{P}}=\left.\left[\phi_{2}\right]\right|_{\mathcal{P}},
$$

then there exists an integer $k>0$ and a unitary $U \in M_{k+1}(Q(B))$ such that

$$
\left\|U^{*} \operatorname{diag}(\phi(a), \sigma(a), \ldots, \sigma(a)) U-\operatorname{diag}(\psi(a), \sigma(a), \ldots, \sigma(a))\right\|<\varepsilon
$$

for all $a \in \mathcal{F}$. 
Proof. We have shown that $Q(B) \in \mathbf{C}_{1,3 \pi, 1}$ in 6.1 Since $Q(\mathcal{K})$ is purely infinite and simple, there are $c(a) \in Q(\mathcal{K})$ with $\|c(a)\| \leq 3$ such that

$$
c(a)^{*} \sigma(a) c(a)=1_{Q(\mathcal{K})}
$$

for $a \in \mathcal{G}^{+}$. Since $Q(\mathcal{K})$ is embedded unitally into $Q(B)$, this implies that $\sigma$ are $(1,3)_{\mathcal{G}^{+}}$-full. Therefore the theorem follows from 5.9

Definition 6.7. Fix a $C^{*}$-algebra, $A$ satisfying the AUCT and $l \geq 1, b \geq \pi$ and $M \geq 1$. Let $\mathcal{F} \subset A$ be a finite subset and let $\varepsilon>0$. Let $\mathcal{G} \subset A$ and $\mathcal{P} \subset \mathbf{P}(A)$ be finite subsets and let $\delta>0$. We say that $(\mathcal{F}, \varepsilon, \mathcal{G}, \mathcal{P}, \delta)$ is a 5 -tuple satisfying requirements in 5.9 if the conclusion of 5.9 holds for any $\mathcal{G}$ - $\delta$-multiplicative contractive completely positive linear maps $\phi, \psi, \sigma: A \rightarrow B$ (with $B \in \mathbf{C}_{l, b, M}$ ) that also satisfy other conditions of $[5.9$ We say $(\mathcal{F}, \varepsilon, \mathcal{G}, \mathcal{P}, \delta)$ is a 5 -tuple satisfying the requirements for the maps in collection $\mathcal{M}$, if the conclusion of 5.9 holds for all $\mathcal{G}$ - $\delta$-multiplicative maps $\phi, \psi$ and $\sigma$ (to a unital $C^{*}$-algebra, $B \in \mathbf{C}_{l, b, M}$ ) in $\mathcal{M}$. This usage is only for the convenience in this paper.

Fix $\mathcal{F}$ and $\varepsilon$ as above. There is a finite subset $\mathcal{P} \subset \mathbf{P}(A)$ such that for any $\mathcal{G}$ and $\delta,(\mathcal{F}, \varepsilon, \mathcal{G}, \mathcal{P}, \delta)$ is a 5 -tuple satisfying the requirements of 5.9 for all homomorphisms (since homomorphisms are multiplicative).

Let $B=A_{1} \oplus A_{2} \oplus \cdots \oplus A_{m}$, where each $A_{i}$ satisfies the AUCT. Fix a finite subset $\mathcal{F}=\mathcal{F}_{1} \oplus \cdots \oplus \mathcal{F}_{m}$, where $\mathcal{F}_{i} \subset A_{i}$. Suppose that $\mathcal{G}_{i} \subset A_{i}$ and $\mathcal{P}_{i} \subset \mathbf{P}\left(A_{i}\right)$ are finite subsets and $\delta_{i}>0$ so that $\left(\mathcal{F}_{i}, \varepsilon, \mathcal{G}_{i}, \mathcal{P}_{i}, \delta_{i}\right)$ is a 5 -tuple satisfying the requirements of 5.9. Let $\delta=\min \left\{\delta_{1}, \ldots, \delta_{m}\right\}, \mathcal{G}=\mathcal{G}_{1} \oplus \cdots \mathcal{G}_{m}$ and $\mathcal{P}=\mathcal{P}_{1} \oplus \cdots \oplus \mathcal{P}_{m}$. Then it is easy to see that $(\mathcal{F}, \varepsilon, \mathcal{G}, \mathcal{P}, \delta)$ is a 5 -tuple satisfying the requirements of 5.9 (for $B)$.

Suppose that $h: A \rightarrow A$ is an automorphism. Then $(h(\mathcal{F}), \varepsilon, h(\mathcal{G}),[h](\mathcal{P}), \delta)$ is a 5 -tuple satisfying the requirements of $[5.9$ if $(\mathcal{F}, \varepsilon, \mathcal{G}, \mathcal{P}, \delta)$ is.

Definition 6.8. Let $A$ be a $C^{*}$-algebra, satisfying the AUCT and let $l \geq 1, b \geq \pi$ and $M \geq 1$ be fixed. Let $B$ be a $\sigma$-unital $C^{*}$-algebra. Let $(\mathcal{F}, \varepsilon, \mathcal{G}, \mathcal{P}, \delta)$ be a 5 -tuple satisfying the requirements in 5.9 (for $\mathbf{C}_{l . b, M}$ ) for all $\mathcal{G}$ - $\delta$-multiplicative contractive completely positive linear maps $\phi$ from $A$ for which $\left.[\phi]\right|_{\mathcal{P}}$ is also well defined. We say a contractive completely positive linear map $\phi: A \rightarrow B$ is a $(\mathcal{F}, \varepsilon, \mathcal{G}, \mathcal{P}, \delta)$-map if it is $\mathcal{G}$ - $\delta$-multiplicative. Note that $\mathcal{G}$ and $\delta$ are used to described the degree of multiplicativity of the map and that it does not require that $B$ belongs to $\mathbf{C}_{l, b, M}$.

Definition 6.9. Let $A$ be a separable $C^{*}$-algebra, which is a generalized inductive limit of $C^{*}$-algebras $A_{n}$ (and write $A=\lim _{n}\left(A_{n}, \phi_{n}\right)$, in the sense of Blackadar and Kirchberg ([BK1] $)$. Suppose that each $A_{n}$ satisfies the AUCT. We use $\phi_{n, \infty}: A_{n} \rightarrow$ $A$ for the contractive completely positive linear map induced by the (generalized) inductive limit. Let $\mathcal{S}_{1}, \mathcal{S}_{2}, \ldots$ be a sequence of finite subsets such that the union is dense in $A$. We say that $A$ satisfies the property $(\mathrm{P})$ if there are finite subsets $\mathcal{F}_{n} \subset A_{k(n)}$ such that $\phi_{k(n), \infty}\left(\mathcal{F}_{n}\right) \supset \mathcal{S}_{n}, \phi_{k(n), k(n)+1}\left(\mathcal{F}_{n}\right) \subset \mathcal{F}_{n+1}$, and $\phi_{k(n), k(n+1)}$ and $\phi_{k(n), \infty}$ are $\left(\mathcal{F}_{n}, \varepsilon_{n}, \mathcal{G}_{n}, \mathcal{P}_{n}, \delta_{n}\right)$-maps with

$$
\left.\phi_{k(n), k(n)+1}\right]\left(\mathcal{P}_{n}\right) \subset \mathcal{P}_{n+1},\left\|\phi_{k(n), k(n)+1}(a)\right\| \geq 1 / 2\|a\|
$$

for all $a \in \mathcal{G}^{+}$and there is $\alpha_{n} \in \operatorname{Hom}_{\Lambda}\left(\underline{K}\left(A_{n}\right), \underline{K}(A)\right)$ such that

$$
\left.\left[\phi_{n, \infty}\right]\right|_{\mathcal{P}_{n}}=\left.\left(\alpha_{n}\right)\right|_{\mathcal{P}_{n}},
$$

where $\sum_{n=k}^{\infty} \varepsilon_{n}<\infty$ and $\sum_{n=1}^{\infty} \delta_{n}<\infty$.

Note that the property (P) depends on the number $l, b$ and $M$. 
Definition 6.10. Let $A$ be a separable $C^{*}$-algebra, and let $\mathcal{C}$ be a class of nuclear $C^{*}$-algebras. Suppose that there is a sequence of $C^{*}$-subalgebras $\left\{A_{n}\right\}$ of $A$ in the class $\mathcal{C}$ such that, for any $\varepsilon>0$ and any finite subset $\mathcal{F} \subset A$, there is $A_{n}$ with

$$
\operatorname{dist}\left(x, A_{n}\right)<\varepsilon \text { for all } x \in \mathcal{F} \text {. }
$$

Then we say $A$ is locally in $\mathcal{C}$.

We say that $A$ is locally AUCT, if $\mathcal{C}$ is the class of nuclear $C^{*}$-algebras which satisfy the AUCT.

The class of $C^{*}$-algebras which are locally in $\mathcal{N}$ will be denoted by $\mathcal{N}_{l}$. It is easy to see that $A$ is locally in $\mathcal{N}_{l}$ must be in $\mathcal{N}_{l}$. Moreover, if $A$ is in $\mathcal{N}_{l}$, then $A$ is locally AUCT.

Proposition 6.11. Every $C^{*}$-algebra, which is locally AUCT satisfies the property (P) (for any $l \geq 1, b \geq \pi$ and $M \geq 1$ ).

Proof. Fix $l \geq 1, b \geq \pi$ and $M \geq 1$. We use $j_{n}: A_{n} \rightarrow A$ for the embedding. Let $\left\{x_{1}, x_{2}, \ldots,\right\}$ be a dense sequence of $A$. Without loss of generality, we may assume that $x_{n} \in A_{n}$. Let $0<\varepsilon_{n}<\min \left\{1 / 2^{n}, \sum_{i=1}^{n-1} \varepsilon_{n}\right\}$. We may assume that $\mathcal{S}_{1}=\left\{x_{1}\right\}=\mathcal{F}_{1}$. Let $\delta_{1}>0$ and let finite sets $\mathcal{G}_{1} \subset A_{1}$ and $\mathcal{P}_{1} \subset \mathbf{P}\left(A_{1}\right)$ be corresponding to $\varepsilon_{1} / 2$ and $\mathcal{F}_{1}$ as required in 5.9. We may assume that $x_{1} \in \mathcal{G}_{1}$. There is $k(2)>1$ and $y_{2} \in A_{k(2)}$ such that

$$
\left\|x_{1}-y_{2}\right\|<\varepsilon_{2} / 2 \text { and } \operatorname{dist}\left(x, A_{k(2)}\right)<\varepsilon_{2} / 2
$$

for all $x \in \mathcal{G}_{1}$.

Let $\mathcal{S}_{2}=\mathcal{G}_{1} \cup\left\{x_{2}\right\}$. Since $A_{k(2)}$ is nuclear, there is a contractive completely positive linear map $L_{1}: A \rightarrow A_{k(2)}$ such that

$$
\left\|L_{1}(a)-a\right\|<\min \left\{\varepsilon_{1} / 2, \delta_{1} / 2\right\}
$$

for $a \in \mathcal{G}_{1}$ (see for example 2.3.12 in [Ln7]).

Let $\phi_{1}=\left.\left(L_{1}\right)\right|_{A_{1}}$. Without loss of generality, we may assume that

$$
\left.\left[\phi_{1}\right]\right|_{\mathcal{P}_{1}}=\left.\left[j_{1}\right]\right|_{\mathcal{P}_{1}} .
$$

Set $\mathcal{F}_{2}=L_{1}\left(\mathcal{G}_{1}\right) \cup\left\{x_{2}\right\}$. Let $\delta_{2}>0$, let finite sets $\mathcal{G}_{2} \subset A_{k(2)}$ and let $\mathcal{P}_{2} \subset$ $\mathbf{P}\left(A_{k(2)}\right)$ be corresponding to $\varepsilon_{2} / 2$ and $\mathcal{F}_{2}$ as required in [5.9. There is $k(3)>k(2)$ and $y_{2}, y_{3} \in A_{k(3)}$ such that

$$
\left\|x_{i}-y_{i}\right\|<\varepsilon_{2} / 2 \text { and } \operatorname{dist}\left(x, A_{k(3)}\right)<\varepsilon_{2} / 2
$$

for all $x \in \mathcal{G}_{2}$.

Let $\mathcal{S}_{3}=\mathcal{G}_{2} \cup\left\{x_{3}\right\}$. Since $A_{k(3)}$ is nuclear, there is a contractive completely positive linear map $L_{2}: A \rightarrow A_{k(3)}$ such that

$$
\left\|L_{2}(a)-a\right\|<\min \left\{\varepsilon_{2} / 2, \delta_{2} / 2\right\}
$$

for $a \in \mathcal{G}_{2}$.

Let $\phi_{2}=\left.\left(L_{2}\right)\right|_{A_{k(2)}}$. Without loss of generality, we may assume that

$$
\left.\left[\phi_{2}\right]\right|_{\mathcal{P}_{2}}=\left.\left[j_{k(2)}\right]\right|_{\mathcal{P}_{2}} .
$$

Continuing this way, we obtain a sequence of maps $\phi_{n}: A_{k(n)} \rightarrow A_{k(n+1)}$. From the construction, we see that $\phi_{n}$ is a $\left(\mathcal{F}_{n}, \varepsilon_{n}, \mathcal{G}_{n}, \mathcal{P}_{n}, \delta_{n}\right)$-map. Set $B=$ $\lim _{n}\left(A_{k(n)}, \phi_{n}\right)$. This is a generalized inductive limit in the sense of Blackadar and Kirchberg $\left([\overline{B K 1})\right.$. Since $j_{n}$ is a monomorphism, $\left[j_{n}\right] \in K K\left(A_{n}, A\right)$. From the above construction, $B$ satisfies the property $(\mathrm{P})$. 
We also have the following approximate intertwining:

$$
\begin{array}{cccccccc}
A_{1} & \stackrel{\phi_{1}}{\longrightarrow} & A_{k(2)} & \stackrel{\phi_{2}}{\longrightarrow} & A_{k(3)} & \stackrel{\phi_{3}}{\longrightarrow} & \cdots \longrightarrow & B \\
\downarrow_{j_{1}} & \nearrow_{L_{1}} & \downarrow_{j_{2}} & \nearrow_{L_{2}} & \downarrow_{j_{3}} & & & \\
A & \stackrel{\mathrm{id}}{\longrightarrow} & A & \stackrel{\mathrm{id}}{\longrightarrow} & A & \stackrel{\mathrm{id}}{\longrightarrow} & \cdots \longrightarrow & A .
\end{array}
$$

Therefore $A \cong B$. So $A$ satisfies the property $(\mathrm{P})$.

Definition 6.12. Let $h: A \rightarrow B$ be a homomorphism. Then $h$ induces homomorphisms from $K_{*}(A, \mathbb{Z} / k \mathbb{Z})$ to $K_{*}(B, \mathbb{Z} / k \mathbb{Z})$. By the naturality, we obtain a homomorphism $\Gamma(h) \in \operatorname{Hom}_{\Lambda}(\underline{K}(A), \underline{K}(B))$. Let $\tau: A \rightarrow Q(B)$ be a trivial extension. There is a monomorphism $\sigma: A \rightarrow M(B \otimes \mathcal{K})$ such that $\pi \circ \sigma=\tau$, where $\pi: M(A \otimes \mathcal{K}) \rightarrow Q(B)$ is the quotient map. Since $K_{*}(M(A \otimes \mathcal{K}))=$ $\{0\}$, we see that $\Gamma(\tau)=0$. Thus, each element in $[\tau] \in \operatorname{Ext}(A, B)$ gives an element $\Gamma(\tau) \in \operatorname{Hom}_{\Lambda}(\underline{K}(A), \underline{K}(Q(B)))$. Therefore we obtain a homomorphism $\Gamma: \operatorname{Ext}(A, B) \rightarrow \operatorname{Hom}_{\Lambda}(\underline{K}(A), \underline{K}(Q(B)))$. Since there is a surjective map from $\operatorname{Hom}_{\Lambda}(\underline{K}(A), \underline{K}(Q(B)))$ to $\operatorname{Hom}\left(K_{*}(A), K_{*-1}(B)\right)$, we also obtain a homomorphism $\gamma: \operatorname{Ext}(A, B) \rightarrow \operatorname{Hom}\left(K_{*}(A), K_{*}(B)\right)$.

Lemma 6.13. Let $A$ be a nuclear $C^{*}$-algebra, satisfying the property (P) (for $\left.\mathbf{C}_{1,3 \pi, 1}\right)$. Then for any $\sigma$-unital $C^{*}$-algebra, the map

$$
\Gamma: \operatorname{Ext}(A, B) \rightarrow \operatorname{Hom}_{\Lambda}(\underline{K}(A), \underline{K}(Q(B)))
$$

is surjective.

Proof. We may also assume that $B$ is stable. Let $\beta \in \operatorname{Hom}_{\Lambda}(\underline{K}(A), \underline{K}(Q(B)))$.

Let $\mathcal{S}_{n}, \mathcal{F}_{n}, \mathcal{G}_{n}, \varepsilon_{n}, \delta_{n}$ and $\alpha_{n} \in \operatorname{Hom}_{\Lambda}\left(\underline{K}\left(A_{n}\right), \underline{K}(A)\right)$ be as in Definition 6.8

Let $\tau_{0}: A \rightarrow Q(B)$ be an absorbing trivial extension (of $A$ by $B$ ) which factors through $B\left(l^{2}\right) \rightarrow Q(\mathcal{K}) \rightarrow Q(B)$. So $\tau_{0}$ is a full monomorphism.

To save notation, without loss of generality, we may assume that $k(n)=n$ in the definition of 6.8 .

Let $\beta_{n} \in \operatorname{Hom}_{\Lambda}\left(\underline{K}\left(A_{n}\right), \underline{K}(Q(B))\right)$ be such that $\beta \circ \alpha_{n}=\beta_{n}$. Since $A_{1}$ satisfies the AUCT, there is a an extension $\tau_{1}: A_{1} \rightarrow Q(B)$ such that $\tau_{1}$ induces $\beta_{1}$. Since $[e] \oplus\left[1_{Q(B)}\right]=[e]$, for any projection $e \in Q(B)$, we may set $L_{1}=\left(\sigma_{1} \oplus \tau_{0}\right) \circ \phi_{1, \infty}$ : $A_{1} \rightarrow Q(B)$. Note that $\Gamma\left(\tau_{0}\right)=0$ and $\tau_{0} \circ \phi_{1, \infty}$ factors through $Q(\mathcal{K})$.

Since $A_{2}$ satisfies the AUCT, there is an extension $\tau_{2}: A_{2} \rightarrow Q(B)$ such that $\tau_{2}$ induces $\beta_{2}$. As above, we set $L_{2}^{\prime}=\tau_{2} \oplus\left(\tau_{0} \circ \phi_{2, \infty}\right): A_{2} \rightarrow Q(B)$. Note that for any integer $m$, there is an isometry $V_{m} \in M_{m}(Q(\mathcal{K})) \subset M_{m}(Q(B))$ such that $V^{*} \tau_{0} V=\tau_{0} \oplus \cdots \oplus \tau_{0}$. So

$$
V^{*} \tau_{0} \circ \phi_{1, \infty} V=\tau_{0} \circ \phi_{1, \infty} \oplus \tau_{0} \circ \phi_{1, \infty} \oplus \cdots \oplus \tau_{0} \circ \phi_{1, \infty} .
$$

Note also that

$$
\tau_{0} \circ \phi_{2, \infty} \circ \phi_{1}=\tau_{0} \circ \phi_{1, \infty} \quad \text { and } \quad L_{2}^{\prime} \circ \phi_{1}=\tau_{2} \circ \phi_{1} \oplus \tau_{0} \circ \phi_{1, \infty} .
$$

Furthermore

$$
\left.\left[\tau_{2} \circ \phi_{1}\right]\right|_{\mathcal{P}_{1}}=\left.\left[\beta \circ \alpha_{2}\right] \circ\left[\phi_{1}\right]\right|_{\mathcal{P}_{1}}=\left.[\beta] \circ\left[\phi_{2, \infty}\right] \circ\left[\phi_{1}\right]\right|_{\mathcal{P}_{1}} .
$$

Thus, by applying [6.6, there is a unitary $U_{1} \in Q(B)$ such that

$$
\operatorname{ad} U_{1} \circ L_{2}^{\prime} \circ \phi_{1} \approx_{1 / 2} L_{1} \text { on } \mathcal{F}_{1} \text {. }
$$


Set $L_{2}=\operatorname{ad} U_{1} \circ L_{2}^{\prime}$. So

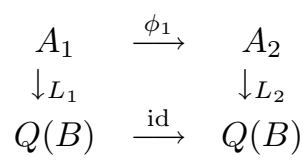

is approximately commutative on $\mathcal{F}_{1}$ within $\varepsilon_{1}$.

We note that we have assumed that $\phi_{n, n+1}\left(\mathcal{F}_{n}\right) \subset \mathcal{F}_{n+1}$.

Continuing this way, since $A_{n+1}$ satisfies the AUCT, there is an extension $\tau_{n+1}$ : $A_{n+1} \rightarrow Q(B)$ such that $\tau_{n+1}$ induces $\beta_{n+1}$. We set $L_{n+1}^{\prime}=\tau_{n} \oplus\left(\tau_{0} \circ \phi_{n, \infty}\right)$ : $A_{n+1} \rightarrow Q(B)$. By the construction ( $\tau_{0}$ is unitarily equivalent to any finite copies of it), applying 6.6 again, there exists a unitary $U_{n} \in Q(B)$ such that

$$
\text { ad } U_{n} \circ L_{n+1}^{\prime} \circ \phi_{n} \approx_{1 / 2^{n}} L_{n} \text { on } S_{n} .
$$

Set $L_{n+1}=\operatorname{ad} U_{n} \circ L_{n+1}^{\prime}$. Hence we obtain an approximate intertwining:

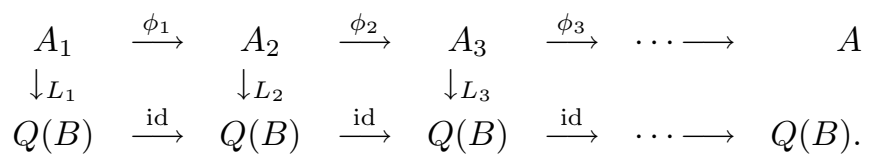

Therefore we obtain the following approximate intertwining:

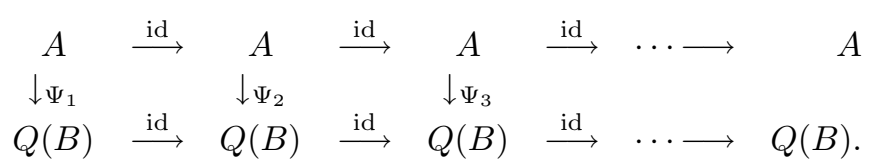

Consequently we obtain, by Elliott's argument, a homomorphism $L: A \rightarrow Q(B)$ induced by the above approximate intertwining. To make sure that $L$ is a monomorphism, set $\tau=L \oplus \tau_{0}$, where $\tau_{0}: A \rightarrow Q(B)$ is an absorbing extension. Since

$$
\left.\left[L_{n}\right]\right|_{\mathcal{P}_{n}}=\left.\beta\right|_{\mathcal{P}_{n}}
$$

it is evident from the construction that $\tau$ induces $\beta$.

Theorem 6.14. If $A$ is a nuclear $C^{*}$-algebra, satisfying the property $(\mathrm{P})$ (for $\left.\mathbf{C}_{1,3,1}\right)$, then the map $\Gamma$ induces an isomorphism

$$
\bar{\Gamma}: K L^{1}(A, B) \rightarrow \operatorname{Hom}_{\Lambda}(\underline{K}(A), \underline{K}(Q(B))) .
$$

Proof. It follows from 6.13 that it suffices to show that $\operatorname{ker} i \Gamma=\mathcal{T}(A, B)$. Let $\tau: A \rightarrow Q(B)$ be an extension. By [4.6, we will show that if $[\tau] \in \operatorname{ker} \Gamma$, then $[\tau] \in \mathcal{T}(A, B)$. Let $\tau_{0}: A \rightarrow Q(B)$ be an absorbing trivial extension. Note again that $\tau_{0}$ is unitarily equivalent to $\tau_{0} \oplus \tau_{0} \oplus \cdots \oplus \tau_{0}$ (for any finitely many $\tau_{0}$ ). It then suffices to show that, for any $\varepsilon>0$ and any finite subset $\mathcal{F} \subset A$, there exists a unitary $U \in Q(B)$ such that

$$
\operatorname{ad} U \circ \tau_{0} \approx_{\varepsilon} \tau \oplus \tau_{0} \text { on } \mathcal{F} .
$$

Let $\tau_{n}=\left.\tau\right|_{A_{n}}$. Since $\Gamma([\tau])=0$, one has $\Gamma\left(\left[\tau_{n}\right]\right)=\Gamma\left(\left[\tau \circ j_{n}\right]\right)=0$. Since $\sigma_{n}=\left.\left(\tau_{0}\right)\right|_{A_{n}}$ are absorbing trivial extensions of $A_{n}$ by $B$, it follows from 6.6 that for any $\varepsilon>0$ and any finite subset $\mathcal{S}_{n} \subset A_{n}$, there is a unitary $W_{n} \in Q(B)$ such that

$$
\text { ad } W_{n} \circ \sigma_{n} \approx_{\varepsilon} \tau_{n} \oplus \sigma_{n} \text { on } \mathcal{S}_{n}
$$

Since $\bigcup_{n} A_{n}$ is dense in $A$, we conclude (from the above) that $\tau \in \mathcal{T}$.

Proposition 6.15. Let $B$ be a $\sigma$-unital $C^{*}$-algebra. Then $\underline{K}(Q(B))=\underline{K}(S B)$. 
Proof. We have the following six-term exact commutative diagram:

$$
\begin{array}{cccc}
K_{0}\left(B \otimes \mathcal{K} \otimes C_{k}\right) & \rightarrow K_{0}\left(M(B \otimes \mathcal{K}) \otimes C_{k}\right) & \rightarrow & K_{0}\left(Q(B) \otimes C_{k}\right) \\
\uparrow & & \downarrow & \downarrow \\
K_{1}\left(Q(B) \otimes \mathcal{K} \otimes C_{k}\right) & \leftarrow K_{1}\left(M(B \otimes \mathcal{K}) \otimes C_{k}\right) & \left.\leftarrow \quad K_{1}(B \otimes \mathcal{K}) \otimes C_{k}\right)
\end{array}
$$

By $[\mathrm{M}], K_{i}\left(M(B \otimes \mathcal{K}) \otimes C_{k}\right)=0(i=0,1)$. Therefore we have

$$
K_{i}(Q(B), \mathbb{Z} / k \mathbb{Z})=K_{i-1}(B, \mathbb{Z} / k \mathbb{Z}) \quad(i=0,1) .
$$

Since $S B \otimes C_{k}=S\left(B \otimes C_{k}\right)$, we have $K_{i}(S B, \mathbb{Z} / k \mathbb{Z})=K_{i-1}(B, \mathbb{Z} / k \mathbb{Z}), i=0,1$.

Theorem 6.16. Let $A$ be a nuclear $C^{*}$-algebra satisfying the property $(\mathrm{P})$. Then $A$ satisfies the AUCT.

Proof. It follows from 6.14 and 6.15 that

$$
K L^{1}(A, B)=\operatorname{Hom}_{\Lambda}(\underline{K}(A), \underline{K}(S B))
$$

and

$$
K L^{0}(A, B)=K L^{1}(A, S B)=\operatorname{Hom}_{\Lambda}(\underline{K}(A), \underline{K}(B)) .
$$

The main result in this section is:

Corollary 6.17. (i) Every $C^{*}$-algebra which is local AUCT satisfies the AUCT.

(ii) Every $C^{*}$-algebra in $\mathcal{N}_{l}$ satisfies the AUCT.

Theorem 6.18. Let $A$ and $B$ be two unital separable simple $C^{*}$-algebras with real rank zero, stable rank one, weakly unperforated $K_{0}(A)$ and with unique tracial states. Suppose also that both $A$ and $B$ are locally type $I$. Then $A \cong B$ if and only if

$$
\left(K_{0}(A), K_{0}(A)_{+},\left[1_{A}\right], K_{1}(A)\right) \cong\left(K_{0}(B), K_{0}(B)_{+},\left[1_{B}\right], K_{1}(B)\right) .
$$

Proof. It follows from [Ln9] that $T R(A)=T R(B)=0$. Since both $A$ and $B$ are locally type I, both $A$ and $B$ are nuclear. Moreover $A, B \in \mathcal{N}_{l}$. Therefore both $A$ and $B$ satisfy the AUCT. Thus 5.10 applies.

Remark 6.19. It should be noted that the class of $C^{*}$-algebras in $\mathcal{N}$ is closed under inductive limits. However, Dadarlat and Eilers in [DE1] gave an example of a $C^{*}$ algebra which is locally homogeneous but it cannot be written as an inductive limit of homogeneous $C^{*}$-algebras. There is no reason to believe, at this moment, that $C^{*}$-algebras locally in $\mathcal{N}_{l}$ are in $\mathcal{N}$. However, here is the question: are there any separable nuclear $C^{*}$-algebras which satisfy the AUCT but not UCT?

\section{AF-EMBEDDING}

This section concerns embeddability of a separable RFD-algebra into a simple AF-algebra. Recall that a $C^{*}$-algebra, $A$ is said to be residually finite dimensional (RFD), if there exists a separating family of finite-dimensional irreducible representations. It was shown in [D] that a nuclear separable RFD algebra which is homotopically dominated by an AF-algebra embeds in an AF-algebra and in [n5] that a nuclear separable RFD algebra satisfying the UCT can be embedded into a (simple) AF-algebra. More recently, among other things, Dadarlat shows ([D3]) that a unital separable nuclear embeddable RFD $C^{*}$-algebra, satisfying the UCT can be embedded into a simple AF-algebra. In this section we consider a sequence of RFD $C^{*}$-algebras. 
Definition 7.1. Let $\left\{A_{n}\right\}$ be a sequence of unital RFD $C^{*}$-algebras. We say that $\left\{A_{n}\right\}$ is asymptotically nuclear and asymptotically satisfies the AUCT, if there are unital injective homomorphisms $h_{n}: A_{n} \rightarrow A_{n+1}$ such that the unital $C^{*}$-algebra $A=\lim _{n}\left(A_{n}, h_{n}\right)$ is nuclear and satisfies the AUCT.

As an application of results of section 2 and the last section, we show that, if $\left\{A_{n}\right\}$ is asymptotically nuclear and asymptotically satisfies the AUCT, then every $A_{n}$ can be embedded into a same unital simple AF-algebra. It is important to note that we do not assume that $A_{n}$ satisfies the UCT nor do we assume that $A_{n}$ is nuclear.

7.2. Let $A=\lim _{n \rightarrow \infty}\left(A_{n}, \phi_{n}\right)$ be a unital nuclear $C^{*}$-algebra, where each $A_{n}$ is a residually finite-dimensional (RFD) $C^{*}$-algebra, and each map $\phi_{n}$ is injective. We also assume that each $A_{n}$ is separable. It is proved in BK1 that every strong NF $C^{*}$-algebra, is such a $C^{*}$-algebra. We may write $A=\overline{\bigcup_{n=1}^{\infty} A_{n}}$, where $A_{n} \subset A_{n+1}$ and each $A_{n}$ is a separable RFD $C^{*}$-algebra. We may further assume that each $A_{n}$ has a unit and that the unit is the same as the unit of $A$. Denote by $\imath_{n}: A_{n} \rightarrow A$ the embedding.

Let $\left\{a_{i, j}\right\}$ be dense sequences of $A_{j}$. Let $\pi_{n, 1}, \ldots, \pi_{n, s(n)}$ be finite-dimensional irreducible representations of $A_{n}$ such that

$$
\max \left\{\left\|\pi_{n, k}\left(a_{i, j}\right)\right\|: 1 \leq k \leq s(n)\right\}>\left\|a_{i, j}\right\|-1 / n\left\|a_{i, j}\right\|
$$

for $1 \leq i, j \leq n$. Without loss of generality, we may assume that $s(n) \geq n$.

Suppose that $\pi_{i, j}$ has rank $r(i, j)$. Let $r(2)=\sum_{i=1}^{s(1)} r(1, i)+1, r(n+1)=$ $n\left(r(n)\left(\sum_{i}^{s(n)} r(n, i)\right)\right)+r(n), n=1,2, \ldots . \quad$ Let $C_{1}^{\prime}=A_{1}, C_{2}^{\prime}=A_{2} \otimes M_{r(2)}, \ldots$, $C_{n}^{\prime}=A_{n} \otimes M_{r(n)}, \ldots$. Define $j_{n, n+1}^{\prime}: C_{n}^{\prime} \rightarrow C_{n+1}^{\prime}$ by

$$
f \mapsto \operatorname{diag}\left(f, \pi_{n, 1} \otimes \operatorname{id}_{r(n)}(f), \pi_{n, 1} \otimes \operatorname{id}_{r(n)}(f), \ldots, \pi_{n, s(n)} \otimes \operatorname{id}_{r(n)}(f)\right),
$$

where $f \in C_{n}^{\prime}$ and $\pi_{n, i} \otimes \mathrm{id}_{r(n)}(f)$ repeats $n$ times. Also, the image of $\pi_{n, i} \otimes \operatorname{id}_{r(n)}$ is in $\left(\mathbb{C} \cdot \operatorname{id}_{A_{n}}\right) \otimes M_{r(n)}$.

Let $C_{1}=A_{1} \otimes Q, \ldots, C_{n}=A_{n} \otimes Q, \ldots$. There is an isomorphism $R_{n}^{\prime}: Q \otimes M_{r(n)} \rightarrow$ $Q$ which gives $\left(R_{n}^{\prime}\right)_{*}: \mathbb{Q} \rightarrow \mathbb{Q}$ by $\left(R_{n}^{\prime}\right)_{*}(r)=\frac{r}{r(n)}$. Set $R_{n}: A_{n} \otimes\left(M_{r(n)} \otimes Q\right) \rightarrow C_{n}$ by $R_{n}(a \otimes b)=a \otimes R_{n}^{\prime}(b)$. Also set $j_{n, n+1}=R_{n+1} \circ\left(j_{n, n+1}^{\prime} \otimes \operatorname{id}_{Q}\right)$.

Let $H_{n}^{\prime}: A_{n} \rightarrow F_{n}$ be defined by

$$
f \mapsto \operatorname{diag}\left(\pi_{n, 1} \otimes \operatorname{id}_{r(n)}(f), \pi_{n, 1} \otimes \operatorname{id}_{r(n)}(f), \ldots, \pi_{n, s(n)} \otimes \operatorname{id}_{r(n)}(f)\right),
$$

where $F_{n}$ is a finite-dimensional $C^{*}$-subalgebra of $M_{r(n+1)}$. Let $H_{n}: C_{n} \rightarrow Q$ be defined by $H_{n}=R_{n+1} \circ\left(H_{n}^{\prime} \otimes \operatorname{id}_{Q}\right)$.

Since $s(n) \geq n$, we compute that

$$
t\left(j_{n, n+1}(1)-H_{n}(1)\right) \leq 1 / n^{2}
$$

for all tracial states $t$ on $C_{n+1}$.

Define $B=\lim _{n}\left(C_{n}, j_{n, n+1}\right)$. In what follows, the map $C_{n} \rightarrow B$ will be denoted by $j_{n, \infty}$.

Similar constructions of this type previously appeared in [Go] and [D2].

Lemma 7.3. $B$ is a unital separable simple $C^{*}$-algebra, with $T R(B)=0$ with divisible $K_{0}(B)$, and with a unique tracial state.

See the proof of Lemma 6 in Ln5] and 4.3 (and 4.4) in [n3]. 
Lemma 7.4. Let $F$ be a nuclear $C^{*}$-algebra, and $A$ be the closure of $\bigcup_{n} A_{n}$, where $A_{n} \subset A_{n+1}, n=1,2, \ldots$ Suppose that there is a contractive completely positive linear map $\phi: F \rightarrow A$. Then, for any $\varepsilon>0$ and any finite subset $\mathcal{F} \subset F$, there is an integer $n>0$ and a contractive completely positive linear map $\psi: F \rightarrow A_{n}$ such that

$$
\|\phi(a)-\psi(a)\|<\varepsilon \quad \text { for all } a \in \mathcal{F} .
$$

Proof. There are (cf. 2.3.13 in [Ln7]) maps $L_{n}: F \rightarrow A_{n}$ for all large $n$ such that $\left\|L_{n}(a)\right\| \leq\|a\|$,

$$
\left\|L_{n}(a)-\phi(a)\right\| \rightarrow 0, \quad \text { as } n \rightarrow \infty .
$$

Define a map $L: F \rightarrow \prod_{n} A_{n}$ by $L(a)=\left\{L_{n}(a)\right\}$ for all $a \in A$. Let $\pi: \prod_{n} A_{n} \rightarrow$ $\prod_{n} A_{n} / \oplus_{n} A_{n}$ be the quotient map. Then $\pi \circ L: F \rightarrow \prod_{n} A_{n} / \oplus_{n} A_{n}$ is a contractive completely positive linear map. Since $F$ is nuclear, by [CE], there is a contractive completely positive linear map $\Psi: F \rightarrow \prod_{n} A_{n}$ such that $\pi \circ \Psi=\pi \circ L$. Write $\Phi(a)=\left\{\psi_{n}(a)\right\}$, where each $\psi_{n}: F \rightarrow A_{n}$ is a contractive completely positive linear map. We have

$$
\left\|\psi_{n}(a)-L_{n}(a)\right\| \rightarrow 0 \quad \text { as } n \rightarrow \infty .
$$

Thus lemma follows.

Corollary 7.5. Let $A$ be a unital separable nuclear $C^{*}$-algebra, which is the closure of the union of increasing sequence $\left\{A_{n}\right\}$. Then for any finite subset $\mathcal{F} \subset A_{n}$ and $\varepsilon>0$, there is an integer $k(n) \geq n$ and a contractive completely positive linear map $L: A \rightarrow A_{k(n)}$ such that

$$
\|L(a)-a\|<\varepsilon \quad \text { for all } a \in A_{n} .
$$

Proof. This follows from 7.4 immediately.

Lemma 7.6. $B$ is nuclear and satisfies the AUCT.

Proof. Let $D=A \otimes Q$. Since $D=\lim _{n \rightarrow \infty} A \otimes M_{n !}, D$ satisfies the AUCT. Let $\mathcal{S}_{1} \subset \mathcal{S}_{2} \subset \cdots$ be a dense sequence of finite subsets of $B$. Without loss of generality, we may assume that $\mathcal{S}_{n} \subset j_{n, \infty}\left(C_{n}\right)$. We may also assume that $\mathcal{S}_{n} \subset \mathcal{S}_{n+1}$. Let $\mathcal{F}_{n} \in C_{n}$ so that $j_{n, \infty}\left(\mathcal{F}_{n}\right)=\mathcal{S}_{n}$. Let $\varepsilon_{n}>0$ so that $\varepsilon_{n}<1 / 2^{n+1}$. Note that $D$ satisfies the AUCT. Let $\mathcal{G}_{n}$ be a finite subset of $D$ and let $\mathcal{P}_{n}$ be a finite subset of $\mathbf{P}(D)$ and $\delta_{n}>0$ be as in [5.9. We may assume, without loss of generality, that $\delta_{n}<\varepsilon_{n}$ and $\sum_{k=n+1}^{\infty} \delta_{k}<\delta_{n}$. By passing to a subsequence if necessary, and with an error no more than $\delta_{n} / 2$, we may assume that $\mathcal{G}_{n} \subset C_{n+1}$.

Let $\eta_{n}>0$. By applying 7.5 and by passing to a subsequence, we may assume that there is a contractive completely positive linear map $L_{n}^{\prime}: A \rightarrow A_{n}$ such that

$$
\left\|L_{n}^{\prime}\left(a_{i, j}\right)-a_{i, j}\right\|<\eta_{n},
$$

$i, j=1,2, \ldots, n$. Set $L_{n}=L_{n}^{\prime} \otimes \operatorname{id}_{Q}$. By choosing sufficiently small $\eta_{n}$, we may assume that

$$
\left\|L_{n}(c)-c\right\|<\delta_{n} / 2
$$

for $c \in \mathcal{F}_{n}$.

Set $\Psi_{n}=\imath_{n+1} \circ j_{n, n+1} \circ L_{n}$. Let $D^{\prime}=\lim _{n}\left(D, \Psi_{n}\right)$ (a generalized inductive limit in the sense of Blackadar and Kirchberg $([\mathrm{BK} 1]))$. From the definition, since each $D$ is nuclear, it is easy to see that $D^{\prime}$ is nuclear. 
Then the following diagram is approximately intertwining:

$$
\begin{array}{clcccccc}
D & \stackrel{\Psi_{1}}{\longrightarrow} & D & \stackrel{\Psi_{2}}{\longrightarrow} & D & \stackrel{\Psi_{3}}{\longrightarrow} & \cdots \longrightarrow & D^{\prime} \\
\downarrow_{L_{1}} & \nearrow_{\imath_{2} \circ j_{1,2}} & \downarrow_{L_{2}} & \nearrow_{\imath_{3} \circ j_{2,3}} & \downarrow_{L_{3}} & & & \\
C_{1} & \stackrel{j_{1,2}}{\longrightarrow} & C_{2} & \stackrel{j_{2,3}}{\longrightarrow} & C_{3} & \stackrel{j_{3,4}}{\longrightarrow} & \cdots \longrightarrow & B .
\end{array}
$$

So, $D^{\prime} \cong B$. We may identify these two $C^{*}$-algebras.

Let $\mathcal{F}_{n}^{\prime}=j_{n, n+1}\left(\mathcal{F}_{n}\right)$. Then, we may write

$$
\mathcal{F}_{n}^{\prime}=R_{n+1}\left(\left\{\operatorname{diag}\left(f, H_{n}^{\prime}(f)\right): f \in \mathcal{F}_{n}\right\}\right),
$$

where $H_{n}^{\prime}: C_{n} \rightarrow M_{\bar{r}(n)} \otimes Q$. Set

$$
\mathcal{P}_{n}^{\prime}=\left[R_{n+1}\right]\left(\mathcal{P}_{n}\right) \oplus\left[H_{n}\right]\left(\mathcal{P}_{n}\right) .
$$

We note that $\left.j_{n, \infty}\right|_{H_{n}\left(\mathcal{F}_{n}\right)}$ is a homomorphism, $M_{\bar{r}(1)} \otimes Q \cong Q$ and $R_{n+1}$ is an automorphism. By [6.7] $\left(\mathcal{F}_{n}^{\prime}, \varepsilon_{n}, \mathcal{G}_{n}^{\prime}, \mathcal{P}_{n}^{\prime}, \delta_{n}\right)$ is a 5 -tuple (for $D$ ) that satisfies the conditions in 6.7. Let $G\left(\mathcal{P}_{n}^{\prime}\right)$ be the group generated by $\mathcal{P}_{n}^{\prime}$. Let $G_{0}=G\left(\mathcal{P}_{n}^{\prime}\right) \cap$ $K_{0}(D)$ and $G_{1}=G\left(\mathcal{P}_{n}^{\prime}\right) \cap K_{1}(D)$. Then $\left[j_{n, \infty}\right]$ gives two homomorphisms from $G_{0}$ to $K_{0}(B)$ and $G_{1}$ to $K_{1}(B)$. Since both $K_{0}(B)$ and $K_{1}(B)$ are divisible, there is $\alpha_{n}^{i}: K_{i}(D) \rightarrow K_{i}(B)$ which extends these two homomorphisms. Since $D$ satisfies the AUCT and both $K_{i}(D)$ and $K_{i}(B)$ are divisible, by the AUCT, $K L(D, B)=$ $\operatorname{Hom}\left(K_{*}(D), K_{*}(B)\right)$. Therefore there is $\alpha_{n} \in K K(D, B)$ such that

$$
\left.\left[j_{n, \infty}\right]\right|_{\mathcal{P}_{n}^{\prime}}=\left.\left(\alpha_{n}\right)\right|_{\mathcal{P}_{n}^{\prime}} .
$$

This shows that $B \cong D^{\prime}=\lim _{n}\left(D, \Psi_{n}\right)$ satisfies the property $(\mathrm{P})$. Hence by 6.16 $B$ satisfies AUCT.

Proposition 7.7. $B$ can be embedded into $C$.

Proof. Since $B$ is a unital separable simple $C^{*}$-algebra, with $T R(B)=0$ and satisfies the AUCT, by $5.11 B$ has the UCT. There is $\alpha_{i}: K_{i}(B) \rightarrow K_{i}(C)$ which is positive and $h\left(\left[1_{B}\right]\right)=\left[1_{C}\right]$. It follows that $B$ is an AH-algebra. It follows from EG] that there is an (injective) homomorphism $h: B \rightarrow C$ such that $h_{* i}=\alpha$.

Since each $A_{n}$ was embedded into $B$, we obtain the following:

Theorem 7.8. Let $\left\{A_{n}\right\}$ be a sequence of RFD $C^{*}$-algebras which is asymptotically nuclear and asymptotically satisfies the UCT. Then every $A_{n}$ can be embedded into a same unital simple AF-algebra.

We note again that we do not assume that $A_{n}$ satisfies the UCT (or AUCT) or that $A_{n}$ is nuclear.

Corollary 7.9. Let $A$ be a unital separable nuclear $R F D C^{*}$-algebra, which satisfies the AUCT. Then A can be embedded into a simple AF-algebra.

Proof. Write $A=\lim _{n \rightarrow \infty}\left(A, \mathrm{id}_{A}\right)$. Then this corollary follows from 7.8

\section{ACKNOWLEDGEMENTS}

We would like to thank Bruce Blackadar who pointed out to us that (6.16) should hold. Part of this work was done when the author was visiting East China Normal University. He thanks the Department of Mathematics for providing a shelter during the summer 2000 in Shanghai. This work was partially supported by the Zhi-Jiang Scholarship (at ECNU), Shanghai Academic Priority Disciplines and a grant from the NSF (US). 


\section{REFERENCES}

[A] W. Arveson, Notes on extensions of $C^{*}$-algebras, Duke Math. J. 44 (1977), 329-355. MF0438137 (55:11056)

[B] B. Blackadar, K-Theory for Operator Algebras, MSRI Monographs, vol. 5, 2nd edition, Cambridge Press, 1998. MR1656031 (99g:46104)

[BK1] B. Blackadar and E. Kirchberg, Generalized inductive limits of finite-dimensional $C^{*}$ algebras Math. Ann. 307 (1997), 343-380. MR.1437044 (98c:46112)

[BK2] B. Blackadar and E. Kirchberg, Inner quasidiagonality and strong NF algebras, Pacific J. Math. 198 (2001), 307-329. MR1835511 (2002f:46103)

[Br] L. G. Brown, The universal coefficient theorem for Ext and quasidiagonality, Proc. International Conf. on Operator Algebras and group Representations (Neptun, Romania, 1980), Monogr. Stud. Math., vol. 17, Pitman, Boston, MA, 1984, pp. 60-64. MR0731763 (85m:46066)

[BrN] N. P. Brown, On the AF embeddability of cross product of AF algebras by the integers, J. Funct. Anal. 160 (1998), 150-175. MR1658700 (2000m:46137)

[CE] M-D. Choi and E. Effros, The completely positive lifting problem for $C^{*}$-algebras, Ann. of Math. 104 (1976), 585-609. MR0417795 (54:5843)

$[\mathrm{Cu}] \quad J$. Cuntz, K-theory for certain $C^{*}$-algebras, Ann. of Math. 113 (1981), 181-197. MF 0604046 (84c:46058)

[D] M. Dadarlat, Residually finite dimensional $C^{*}$-algebras, Cont. Math., Amer. Math. Soc. 228 (1998), 45-50. MF,1667653 (99m:46133)

[D2] M. Dadarlat, Nonnuclear subalgebras of AF algebras, Amer. J. Math. 122 (2000), 581-597. MF 1759889 (2001g:46141)

[D3] M. Dadarlat, Embeddings of nuclear embeddable $C^{*}$-algebras, preprint 2000.

[DE1] M. Dadarlat and S. Eilers, On the classification of nuclear $C^{*}$-algebras, Proc. London Math. Soc. 85 (2002), 168-210. MR.1901373 (2003d:19006)

[DE2] M. Dadarlat and S. Eilers, Asymptotic unitary equivalence in KK-theory, J. Reine Angew. Math. 507 (1999), 1-13. MR.1670254 (2000b:46094)

[DL1] M. Dadarlat and T. Loring, K-homology, asymptotic representations and unsuspended E-theory, J. Funct. Anal. 126 (1994), 367-383. MR1305073 (96d:46092)

[DL2] M. Dadarlat and T. Loring, A universal multi-coefficient theorem for the Kasparov groups, Duke Math. J. 84 (1996), 355-377. MR 1404333 (97f:46109)

[El11] G. A. Elliott, On the classification of $C^{*}$-algebras of real rank zero, J. Reine Angew. Math. 443 (1993), 179-219. MR1241132 (94i:46074)

[EG] G. A. Elliott and G. Gong, On the classification of $C^{*}$-algebras of real rank zero, II, Ann. Math. 144 (1996), 497-610. MR.1426886 (98j:46055)

[GL1] G. Gong and H. Lin, Almost multiplicative morphisms and almost commuting matrices, J. Operator Theory 40 (1998), no. 2, 217-275. MF.1660385 (2000c:46105)

[GL2] G. Gong and H. Lin, Almost Multiplicative Morphisms and K-Theory, Inter. J. Math. 8 (2000), 983-1000. MR 1797674 (2001j:46081)

[Go] K. R. Goodearl, Notes on a class of simple $C^{*}$-algebras with real rank zero, Publ. Mat. 36 (1992), no. 2A, 637-654. MR,1209829 (94f:46092)

$[\mathrm{H}] \quad$ N. Higson, A characterization of $K K$-theory, Pacific J. Math. 126 (1987), 253-276. MF 0869779 (88a:46083)

[Ka] G. Kasparov, Hilbert $C^{*}$-modules: theorems of Stinespring and Voiculescu, J. Operator Theory 4 (1980), 133-150. MR0587371 (82b:46074)

[K1] E. Kirchberg, On non-semisplit extensions, tensor products and exactness of group $C^{*}$ algebras, Invent. Math. 112 (1993), 39-77. MR1218321 (94d:46058)

[K2] E. Kirchberg, The classification of purely infinite simple $C^{*}$-algebras using Kasparov's theory, to appear in the Fields Institute Communication series.

[KP] E. Kirchberg and N. C. Phillips, Embedding of exact $C^{*}$-algebras into $\mathcal{O}_{2}$, J. Reine und Angew. Math. 552 (2000), 17-53. MR1780426 (2001d:46086a)

[Ln1] H. Lin, Classification of simple $C^{*}$-algebras with unique traces, Amer. J. Math. 119 (1997), 1263-1289. MR 1657178 (2000c:46106)

[Ln2] H. Lin, Stable approximate unitary equivalences of homomorphisms, J. Operator Theory 47 (2002), 343-378. MR1911851 (2003c:46082) 
[Ln3] H. Lin, Tracially AF $C^{*}$-algebras, Trans. Amer. Math. Soc. 353 (2001), 693-722. MF 1804513 (2001j:46089)

[Ln4] H. Lin, Classification of simple TAF $C^{*}$-algebras, Canad. J. Math. 53 (2001), 161-194. MF 1814969 (2002h:46102)

[Ln5] H. Lin, Residually finite dimensional and AF-embeddable $C^{*}$-algebras, Proc. Amer. Math. Soc. 129 (2001), 1689-1696. MR1814098 (2002g:46099)

[Ln6] H. Lin, Tracial topological rank of $C^{*}$-algebras, Proc. London Math. Soc. 83 (2001), 199234. MR 1829565 (2002e:46063)

[Ln7] H. Lin, An introduction to the classification of amenable $C^{*}$-algebras, World Scientific, New Jersey/London/Singapore/Hong Kong/ Bangalore, 2001. MR1884366 (2002k:46141)

[Ln8] H. Lin, Classification of simple $C^{*}$-algebras with tracial topological zero, Duke Math. J., to appear.

[Ln9] H. Lin, Locally type I simple $C^{*}$-algebras, preprint 1998.

[Lo1] T. Loring, $C^{*}$-algebras generated by stable relations, J. Funct. Anal. 112 (1993), 159-203. MF $1207940(94 \mathrm{k}: 46115)$

[Lo2] T. Loring, Stable relations. II. Corona semiprojectivity and dimension-drop $C^{*}$-algebras, Pacific J. Math. 172 (1996), no. 2, 461-475. MR1386627 (97c:46070)

[M] J. Mingo, $K$-theory and multipliers of stable $C^{*}$-algebras, Trans. Amer. Math. Soc. 299 (1987), 397-411. MF0869419 (88f:46136)

[Ped1] G. K. Pedersen, $S A W^{*}$-algebras and corona $C^{*}$-algebras, contributions to noncommutative topology. J. Operator Theory 15 (1986), 15-32. MR0816232 (87a:46095)

[Ped] G. K. Pedersen, $C^{*}$-algebras and their automorphism groups, Academic Press, London, 1979. MR0548006 (81e:46037)

[Pi] M. Pimsner, Embedding some transformation group $C^{*}$-algebras into AF algebras, Ergod. Th. Dynam. Sys. 3 (1983), 613-626. MR0753927 (86d:46054)

[P1] N. C. Phillips, Approximation by unitaries with finite spectrum in purely infinite $C^{*}$ algebras, J. Funct. Anal. 120 (1994), 98-106. MF 1262248 (95c:46092)

[P2] N. C. Phillis, A classification theorem for nuclear purely infinite simple $C^{*}$-algebras, Doc. Math. 5 (2000), 49-114. MF 1745197 (2001d:46086b)

[Rr1] M. Rørdam, Classification of inductive limits of Cuntz algebras, J. Reine Angew. Math. 440 (1993), 175-200. MR.1225963 (94k:46120)

[Rr2] M. Rørdam, Classification of certain infinite simple $C^{*}$-algebras, J. Funct. Anal. 131 (1995), 415-458. MF1345038 (96e:46080a)

[RS] J. Rosenberg and C. Schochet, The Kunneth theorem and the universal Coefficient theorem for Kasparov's generalized functor, Duke Math. J. 55 (1987), 431-474. MR0894590 (88i:46091)

[S1] C. Schochet, Topological methods for $C^{*}$-algebras. III, Axiomatic homology, Pacific J. Math. 114 (1984), 399-445. MR0757510 (86g:46102)

[S2] C. Schochet, Topological methods for $C^{*}$-algebras IV, mod $p$ homology, Pacific J. Math. 114 (1984), 447-468. MR.0757511 (86g:46103)

[S3] C. L. Schochet, The fine structure of the Kasparov groups I: Continuity of the KK-paring, J. Funct. Anal. 186 (2001), 25-61. MR1863291 (2003d:19009)

[S4] C. L. Schochet, The fine structure of the Kasparov groups II: Topologizing the UCT, J. Funct. Anal. 194 (2002), 263-287. MR1934604 (2003k:19007)

[Sp] J. S. Spielberg, Embedding $C^{*}$-algebra, extensions into AF-algebras, J. Funct. Anal. 81 (1988), 325-344. MF0971884 (90a:46154)

[Vo] D. Voiculescu, Almost inductive limit automorphisms and embedding into AF-algebras, Ergod. Th. Dynam. Sys. 6 (1986), 475-484. MR0863206 (88k:46073)

[Zh1] S. Zhang, On the structure of projections and ideals of corona algebras, Canad. J. Math. 41 (1989), 721-742. MR1012625 (90h:46094)

[Zh2] S. Zhang, On the exponential rank and exponential length of $C^{*}$-algebras, J. Operator Theory 28 (1992), 337-355. MR.1273050 (95d:46065)

Department of Mathematics, East China Normal University, Shanghai, People's RePUBlic OF China

Current address: Department of Mathematics, University of Oregon, Eugene, Oregon 974031222 